\title{
New $\mathbf{V}_{\mathrm{e}}+\overline{\mathbf{v}}_{\mathrm{e}}$ appearance results from NOvA
}

\author{
Bruce Howard | Indiana University
}

This document was prepared by [NOvA Collaboration] using the resources of the Fermi National Accelerator Laboratory (Fermilab), a U.S. Department of Energy, Office of Science, HEP User Facility.

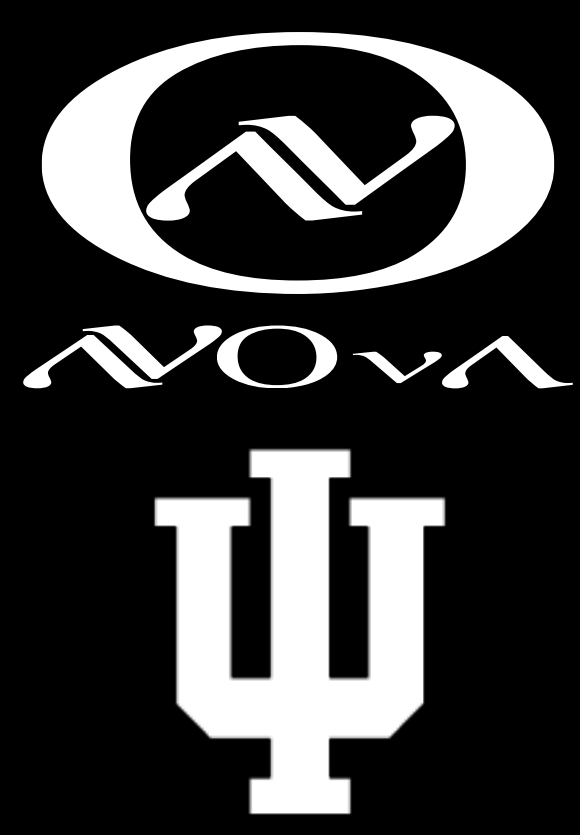

New Perspectives 2018

19 June 2018 


\section{Context}

- Many details in talk from J. Vasel

- Functionally-similar near and far detectors separated by $810 \mathrm{~km}$

- In addition to $v_{\mu}$ disappearance, can study appearance $v_{\mu} \rightarrow v_{e}\left(\bar{V}_{\mu} \rightarrow \bar{v}_{e}\right)$ to probe 3-flavor neutrino oscillations

- Mixing angles and octant sensitivity?

- Mass splitting and hierarchy?

- CP violation?

- Updated NOvA analysis with, for the first time, antineutrino beam data

- Antineutrino dataset $6.9 \times 10^{20}$ protons on target (neutrino dataset $8.9 \times 10^{20}$ fulldetector equivalent protons on target)

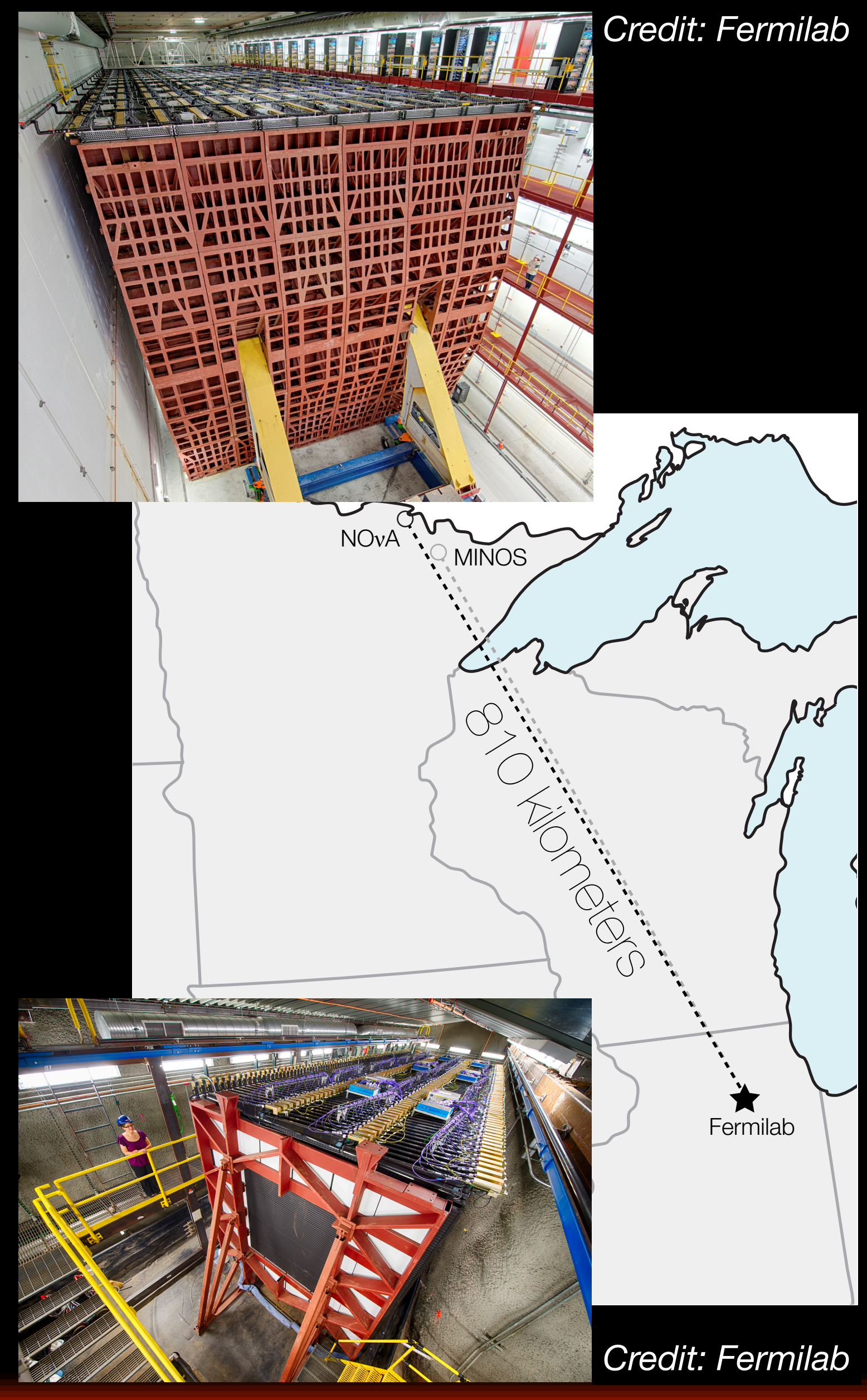




\section{Event selection for $v_{e}+\bar{v}_{e}$}

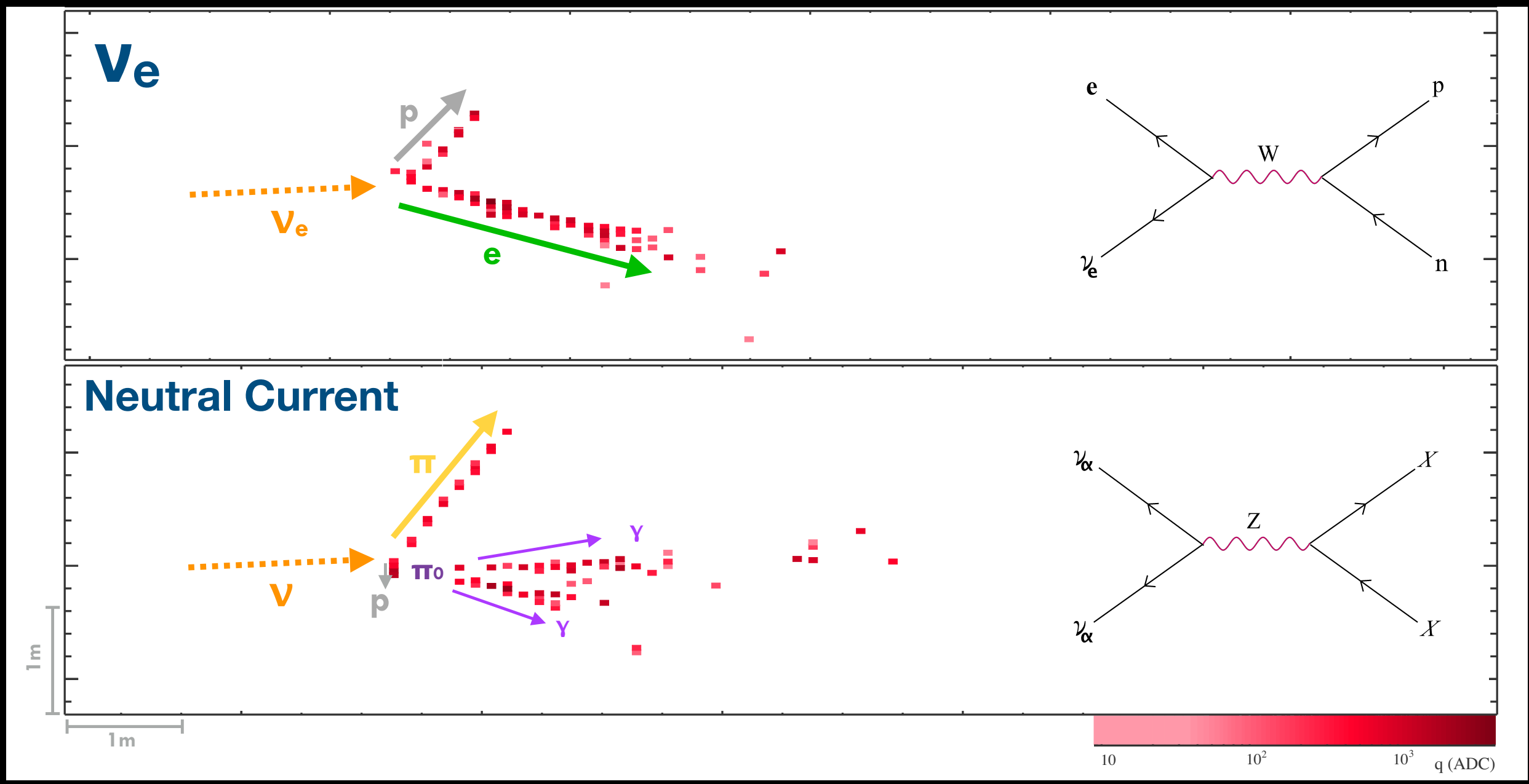

- Details of reconstruction detailed in talk by M. Groh

- Select events $\mathrm{W} / \mathrm{V}_{\mathrm{e}}\left(\overline{\mathrm{V}}_{\mathrm{e}}\right)$ classifier score \& reconstructed information indicating likely $\mathrm{v}_{\mathrm{e}}\left(\overline{\mathrm{V}}_{\mathrm{e}}\right)$ candidate

- NOvA uses CVN (Convolutional Visual Network): event classification based on final state topologies

- Selections broken into two subcategories based on CVN electron score

- Far detector: extra peripheral sample w/ larger cosmic background but recovers appearance signal - Energy range of interest 1-4 GeV in far detector (0-4.5 GeV in near detector, peripheral) 


\section{Predicting far detector spectra}

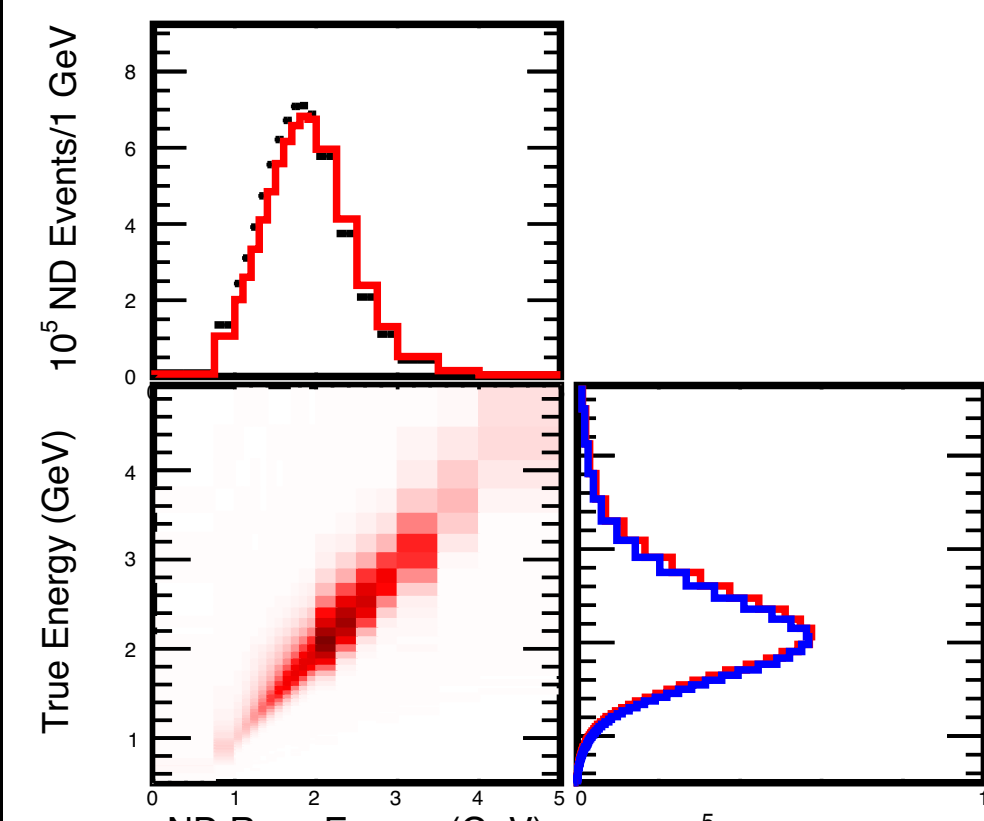

ND Reco Energy (GeV)
$10^{5}$ ND Events
ND data

- Base Simulation

Data-Driven Prediction

- Functionally similar near, far detectors: extrapolation reduces overall systematics (reduction factor 2)

- Near detector $v_{\mu}$ spectrum extrapolated to far detector given oscillation parameters forms appearance signal prediction

- Expect fewer signal events in antineutrino beam than in neutrino beam: (Flux $\otimes$ Cross-section) reduced for antineutrinos and somewhat less exposure in antineutrino beam

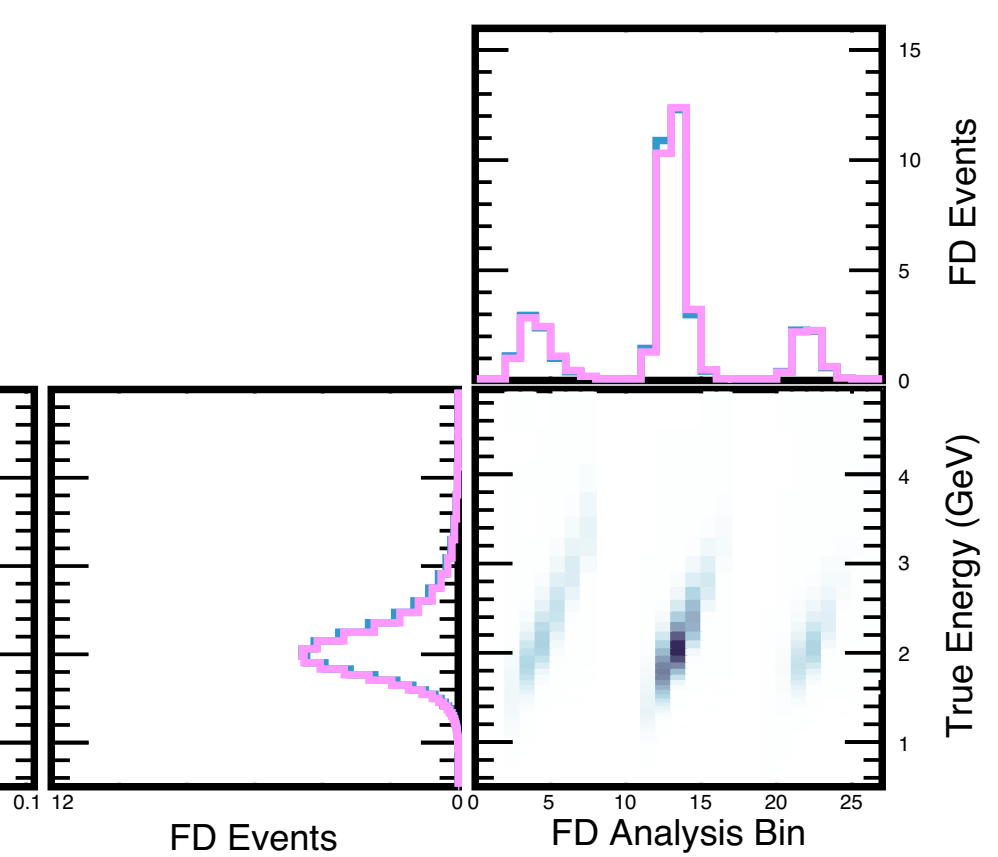

$10^{-3}$ F/N Ratio $\quad \mathrm{P}\left(v_{\mu} \rightarrow v_{\mathrm{e}}\right)$ FD Events
$\mathrm{N}$

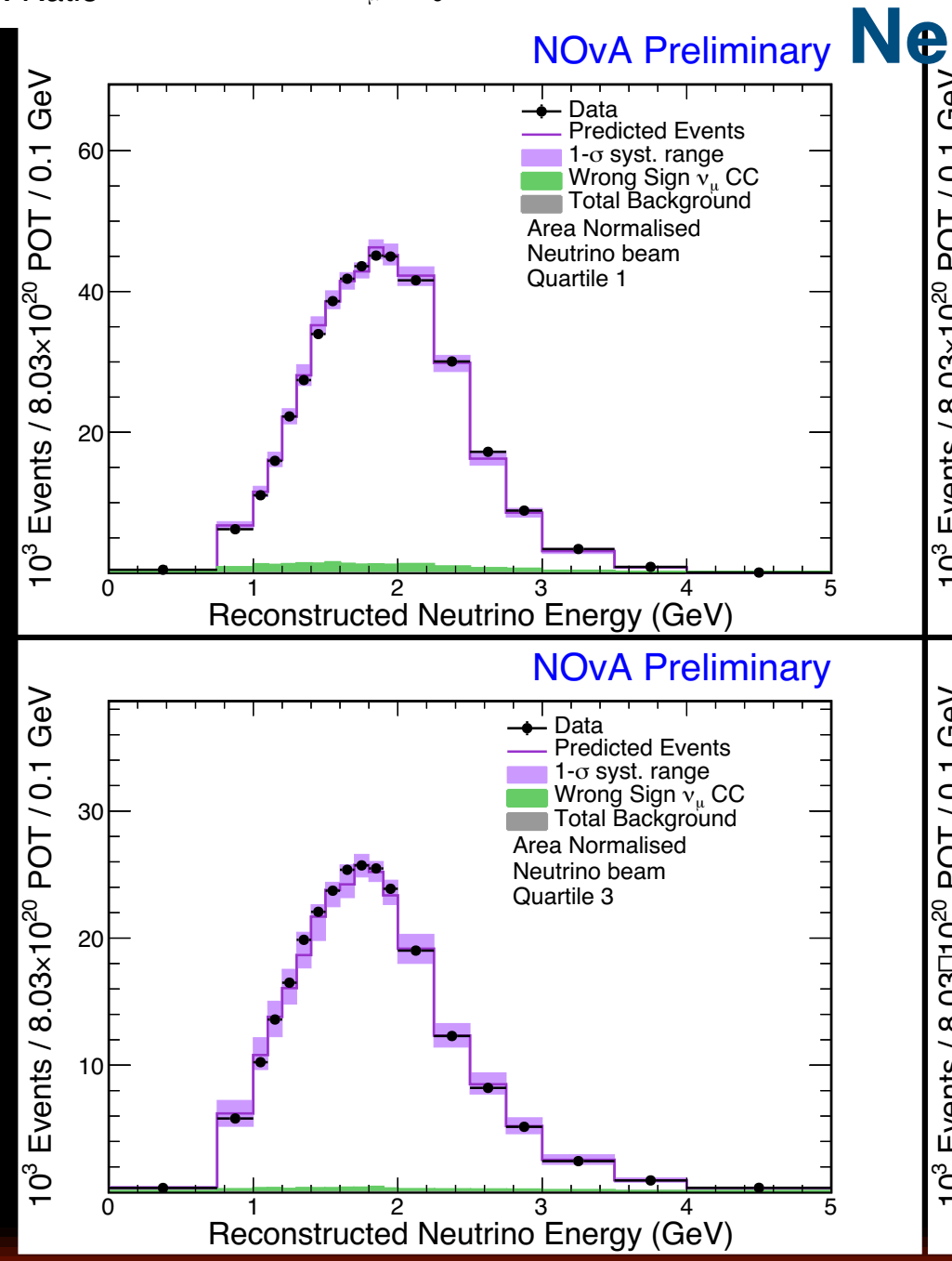

Reconstructed Neutrino Energy (GeV)

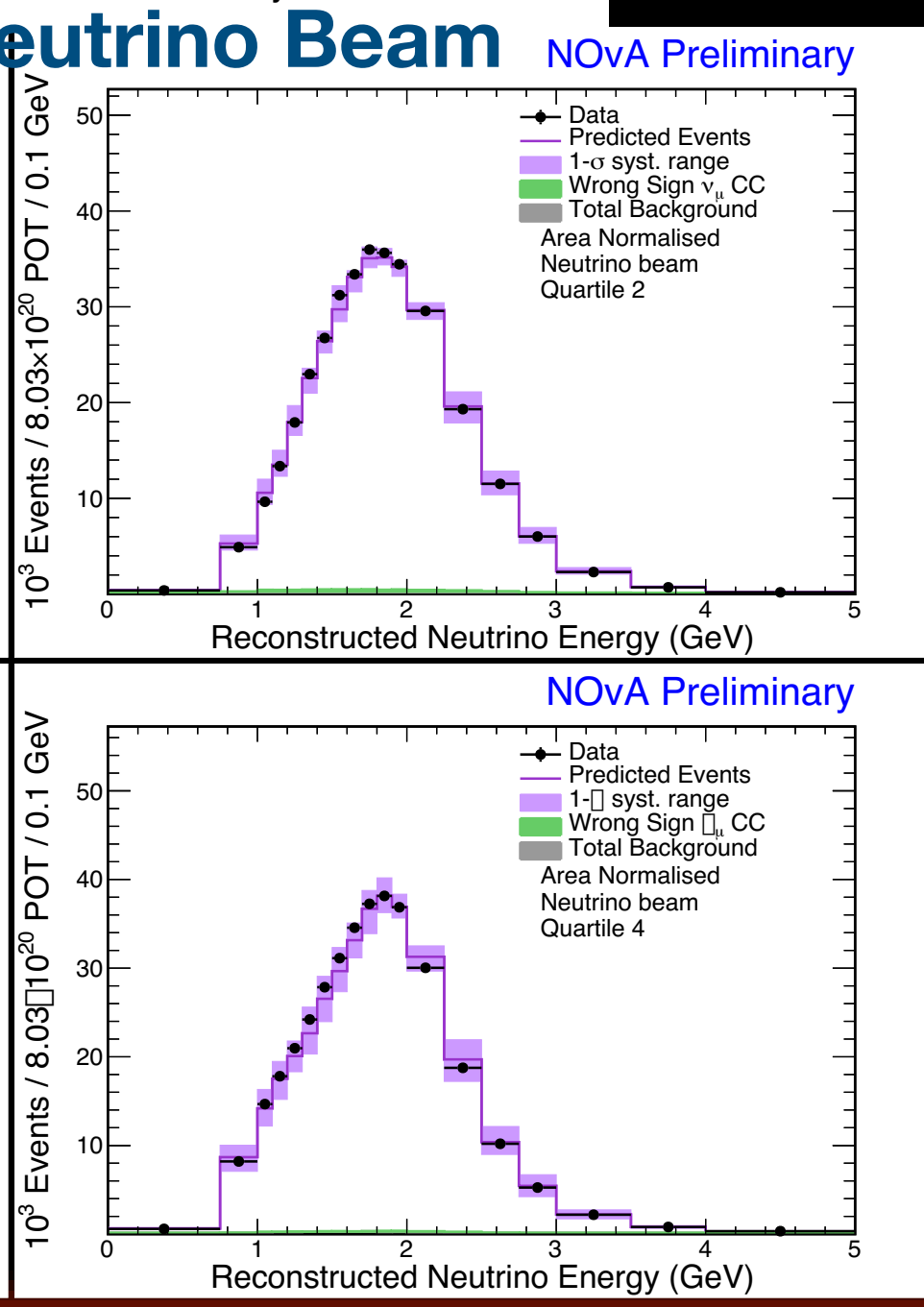

B. Howard 


\section{Near Detector Background}

- Selected near detector candidate spectra contain only backgrounds for appearance analysis: inform far detector background prediction via far/near ratio

- Components constrained via datadriven methods

- In antineutrino beam, for now, data/ MC differences are scaled proportionally in each energy bin to the components.

- In neutrino beam, with higher stats, more refined method used which examines components separately.

- Decomposed ND spectra agree w/ MC by construction

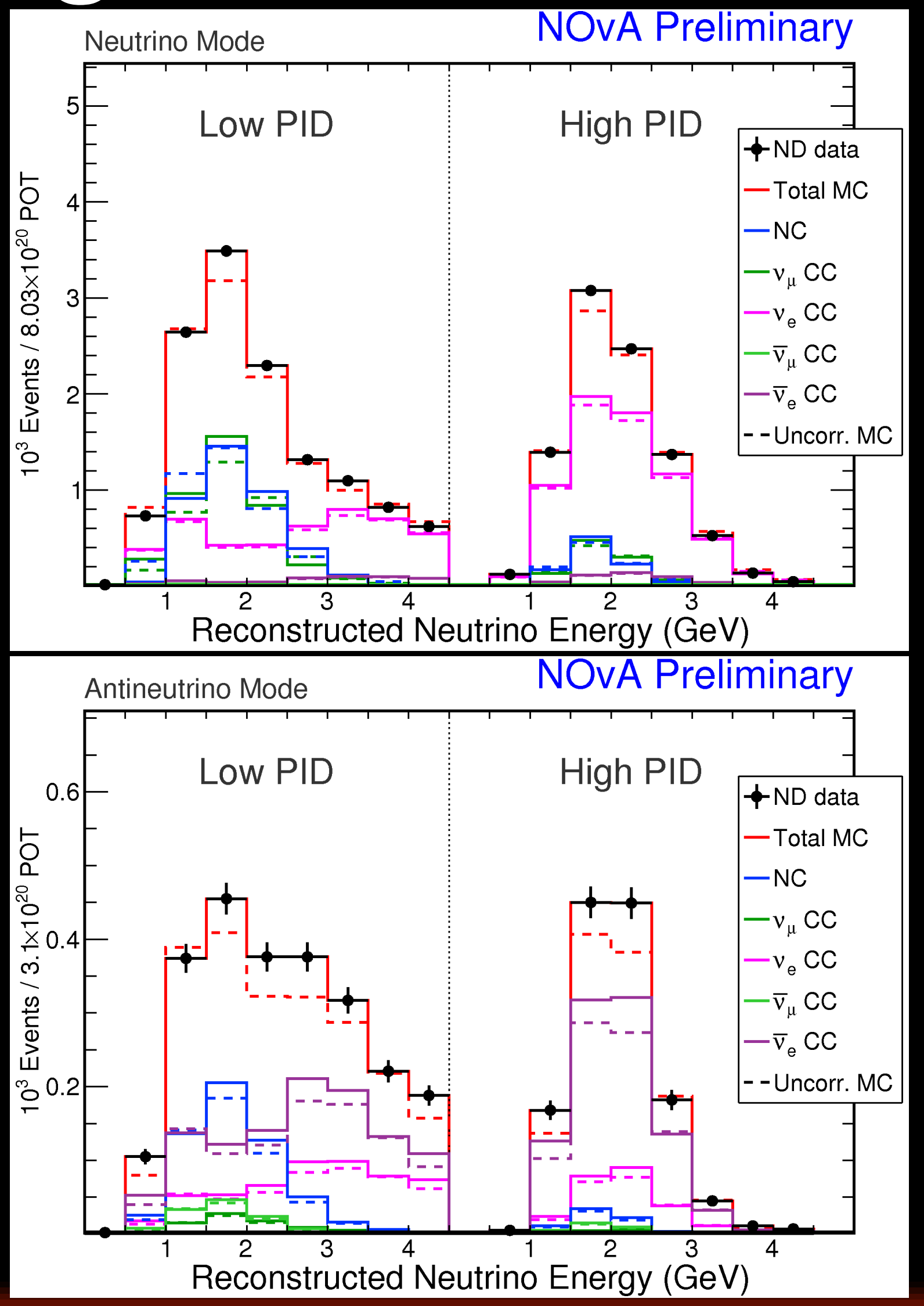




\section{Near Detector Background}

- Wrong-sign ( $\mathrm{v}$ in $\overline{\mathrm{v}}$ beam) fraction estimate in near detector $\overline{\mathrm{V}}_{\mu}$ selection $11 \%$, checked w/ neutron capture rates

- Oscillates to becomes appearance background

- Wrong-sign fraction estimate in near detector beam ' $\overrightarrow{\mathrm{v}}$ e selected background $22 \%$ for antineutrino beam in higher (purer in ' $\vec{v}_{e}^{\prime}$ ) CVN sample. Check w/ identified protons \& event kinematics
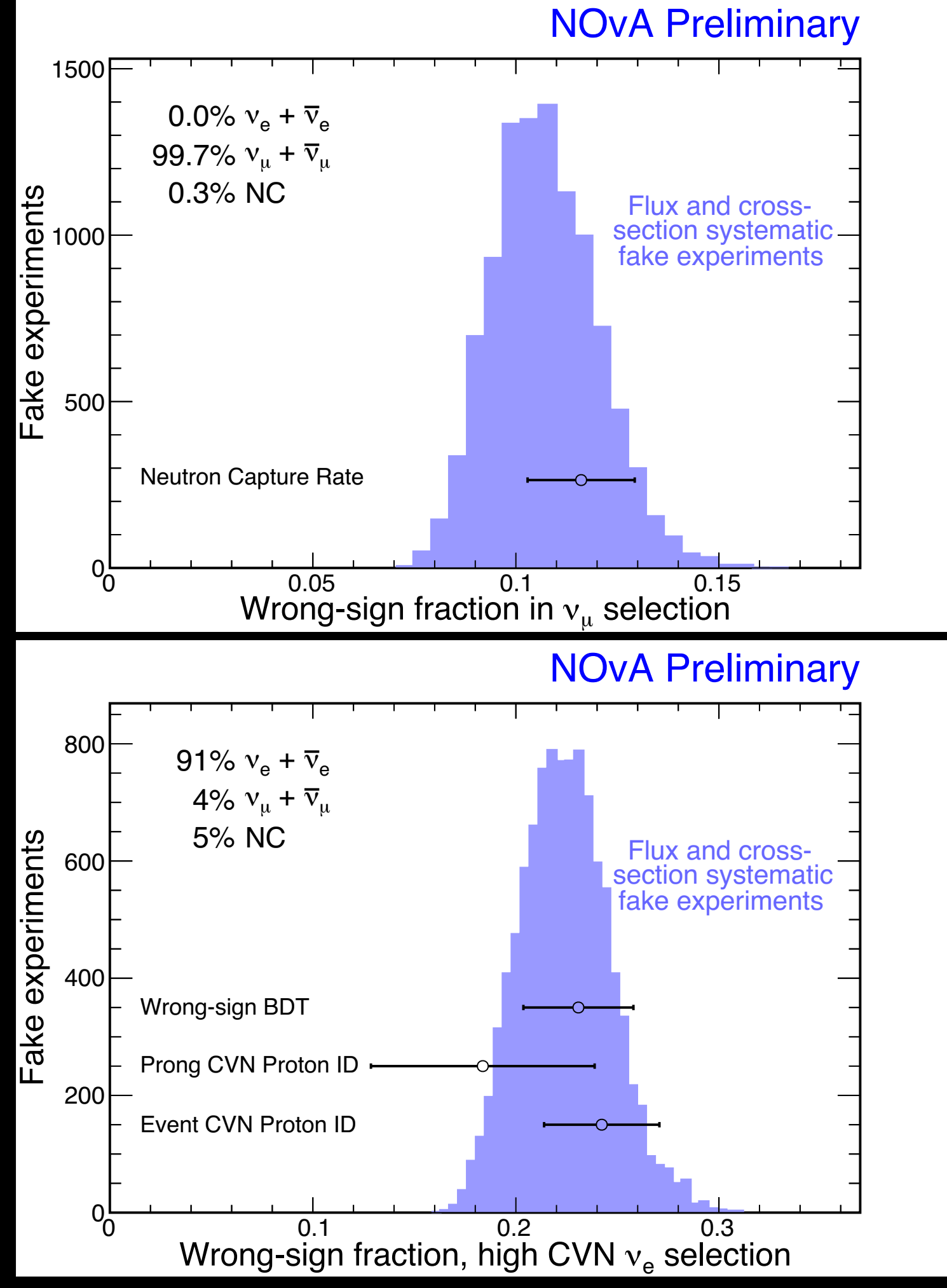


\section{Predicting far detector spectra}

- External constraint on $\sin ^{2} 2 \Theta_{13}$ from PDG average

- Enhancement/suppression due to matter effect based on mass hierarchy. For normal hierarchy $(\mathrm{NH})$, matter effect enhances $v_{e}$, suppresses $\bar{v}_{e}$

- Inverted Hierarchy (IH) opposite

- Oscillation parameters determine further $v_{e}, \bar{v}_{e}$ enhancement/suppression (overall and relative)

- E.g. for $\delta_{\mathrm{C} P}=\pi / 2$, IH: suppression of $v_{e}$ candidates relative to $\bar{v}_{e}$

- Alternatively, large suppression of $\bar{v}_{e}$ relative to $v_{e}$ for $\delta_{C P}=3 \pi / 2, N H$

- Predictions range from 10-22 $\overline{\mathrm{v}}_{\mathrm{e}}$

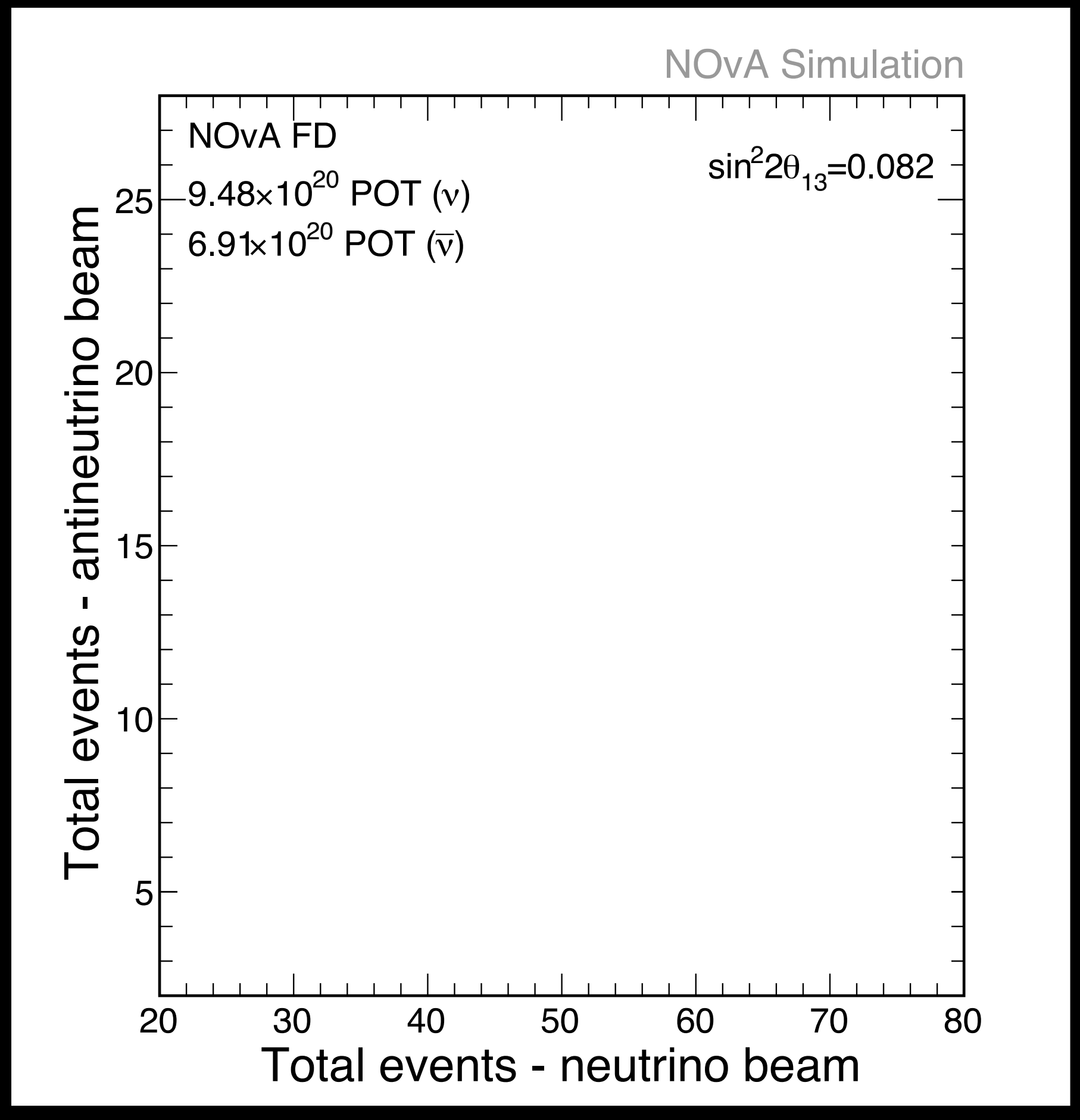
candidates, 30-75 $v_{\mathrm{e}}$ candidates 


\section{Predicting far detector spectra}

- External constraint on $\sin ^{2} 2 \Theta_{13}$ from PDG average

- Enhancement/suppression due to matter effect based on mass hierarchy. For normal hierarchy $(\mathrm{NH})$, matter effect enhances $v_{e}$, suppresses $\bar{v}_{e}$

- Inverted Hierarchy (IH) opposite

- Oscillation parameters determine further $v_{e}, \bar{v}_{e}$ enhancement/suppression (overall and relative)

- E.g. for $\delta_{\mathrm{CP}}=\pi / 2$, IH: suppression of $v_{e}$ candidates relative to $\bar{v}_{e}$

- Alternatively, large suppression of $\bar{v}_{e}$ relative to $v_{e}$ for $\delta_{C P}=3 \pi / 2, N H$

- Predictions range from $10-22 \bar{v}_{\mathrm{e}}$

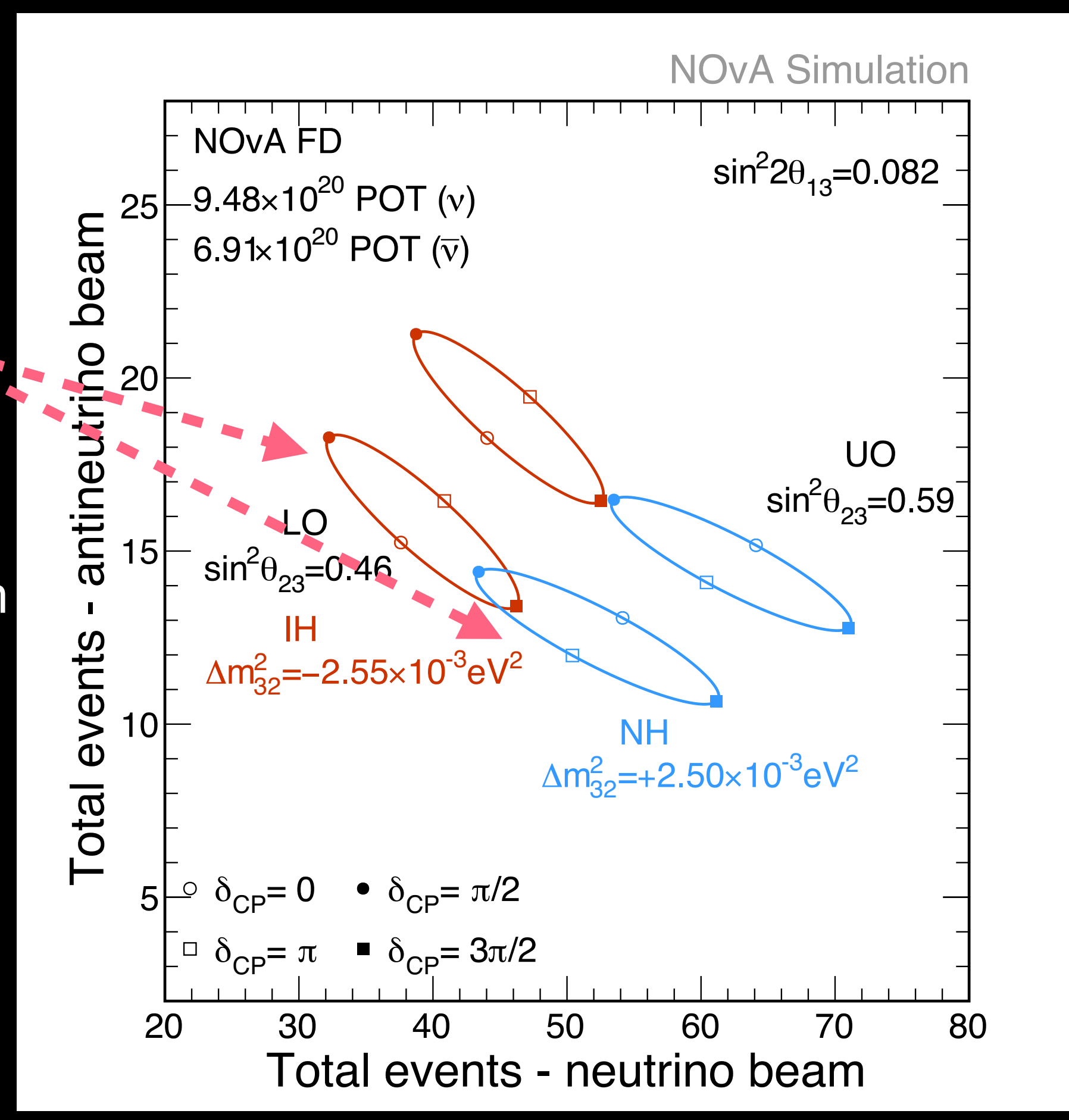
candidates, 30-75 $v_{\mathrm{e}}$ candidates 


\section{Predicting far detector spectra}

- External constraint on $\sin ^{2} 2 \Theta_{13}$ from PDG average

- Enhancement/suppression due to matter effect based on mass hierarchy. For normal hierarchy $(\mathrm{NH})$, matter effect enhances $v_{e}$, suppresses $\bar{v}_{e}$

- Inverted Hierarchy (IH) opposite

- Oscillation parameters determine further $v_{e}, \bar{v}_{e}$ enhancement/suppression (overall and relative)

- E.g. for $\delta_{\mathrm{CP}}=\pi / 2$, IH: suppression of $v_{e}$ candidates relative to $\bar{v}_{e}$

- Alternatively, large suppression of $\bar{v}_{e}$ relative to $v_{e}$ for $\delta_{C P}=3 \pi / 2, N H$

- Predictions range from $10-22 \bar{v}_{\mathrm{e}}$

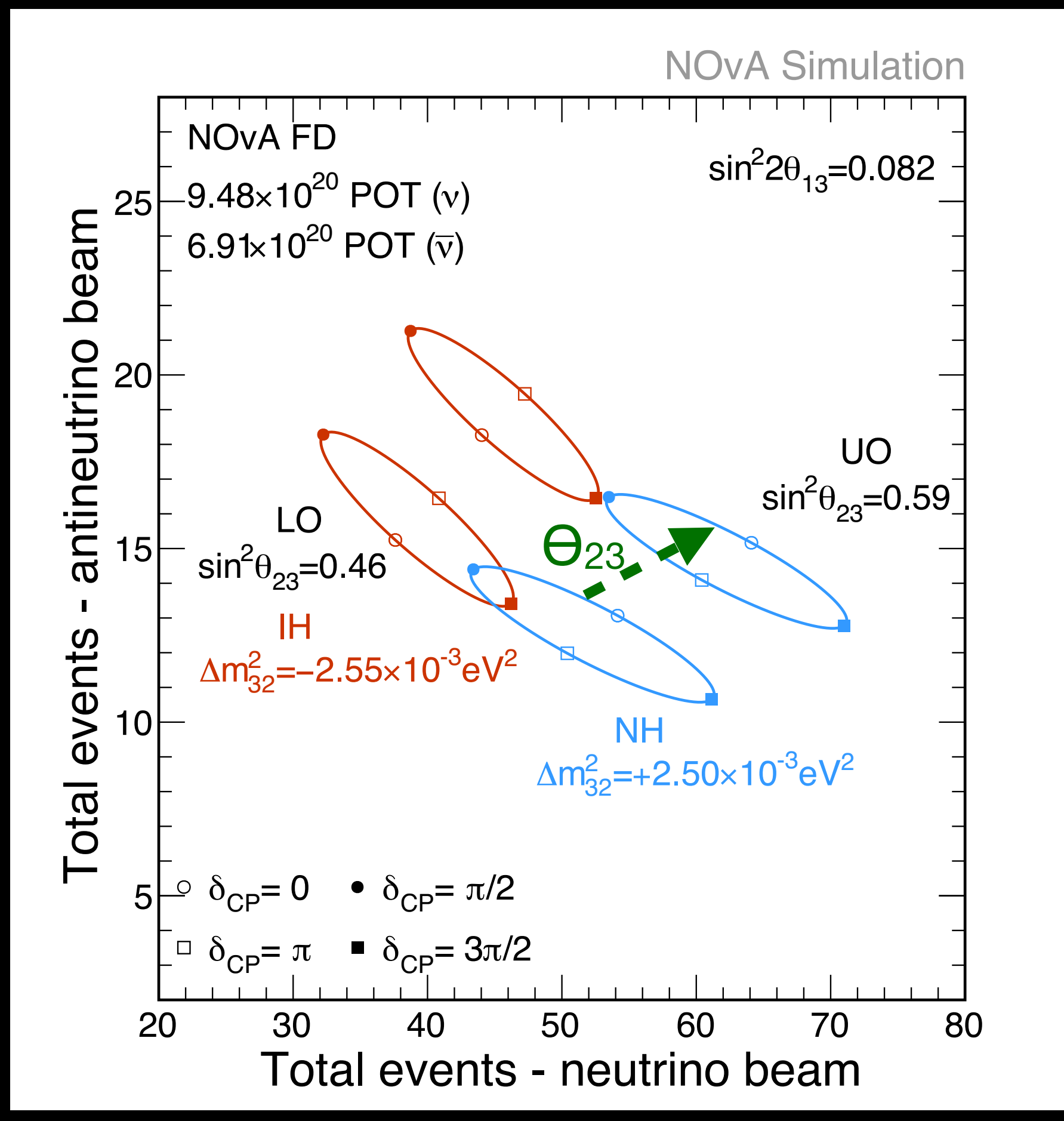
candidates, 30-75 $v_{\mathrm{e}}$ candidates 


\section{Predicting far detector spectra}

- External constraint on $\sin ^{2} 2 \Theta_{13}$ from PDG average

- Enhancement/suppression due to matter effect based on mass hierarchy. For normal hierarchy $(\mathrm{NH})$, matter effect enhances $v_{e}$, suppresses $\bar{v}_{e}$

- Inverted Hierarchy (IH) opposite

- Oscillation parameters determine further $v_{e}, \bar{v}_{e}$ enhancement/suppression (overall and relative)

- E.g. for $\delta_{\mathrm{CP}}=\pi / 2$, IH: suppression of $v_{e}$ candidates relative to $\bar{v}_{e}$

- Alternatively, large suppression of $\bar{v}_{e}$ relative to $v_{e}$ for $\delta_{C P}=3 \pi / 2, N H$

- Predictions range from $10-22 \bar{v}_{\mathrm{e}}$

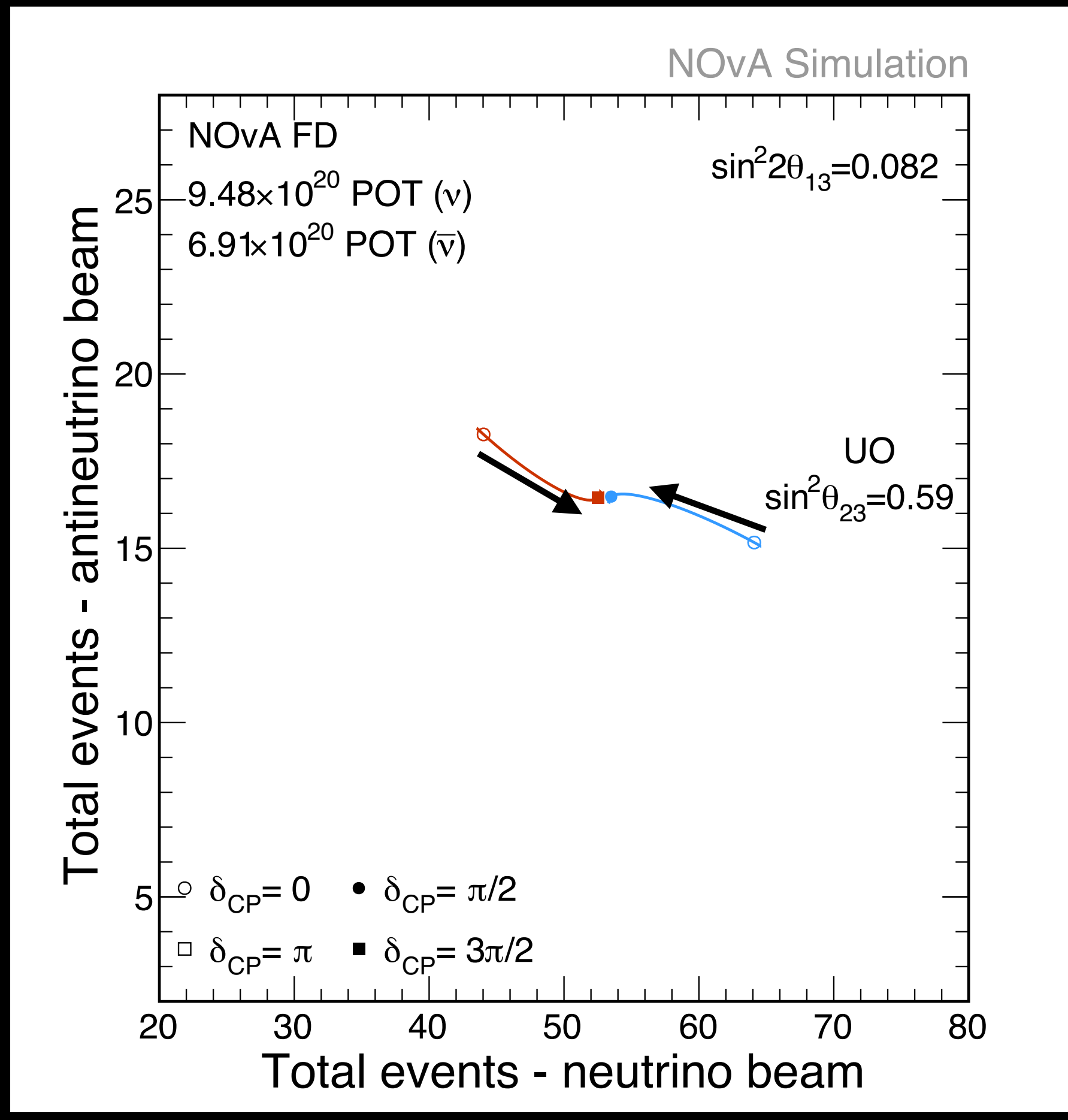
candidates, 30-75 $v_{\mathrm{e}}$ candidates 


\section{Predicting far detector spectra}

- External constraint on $\sin ^{2} 2 \Theta_{13}$ from PDG average

NOvA Simulation

- Enhancement/suppression due to matter effect based on mass hierarchy. For normal hierarchy $(\mathrm{NH})$, matter effect enhances $v_{e}$, suppresses $\bar{v}_{e}$

- Inverted Hierarchy (IH) opposite

- Oscillation parameters determine further $v_{e}, \bar{v}_{e}$ enhancement/suppression (overall and relative)

- E.g. for $\delta_{\mathrm{CP}}=\pi / 2$, IH: suppression of $v_{e}$ candidates relative to $\bar{V}_{e}$

- Alternatively, large suppression of = $\bar{v}_{e}$ relative to $v_{e}$ for $\delta_{C P}=3 \pi / 2, N H$

- Predictions range from $10-22 \bar{v}_{\mathrm{e}}$

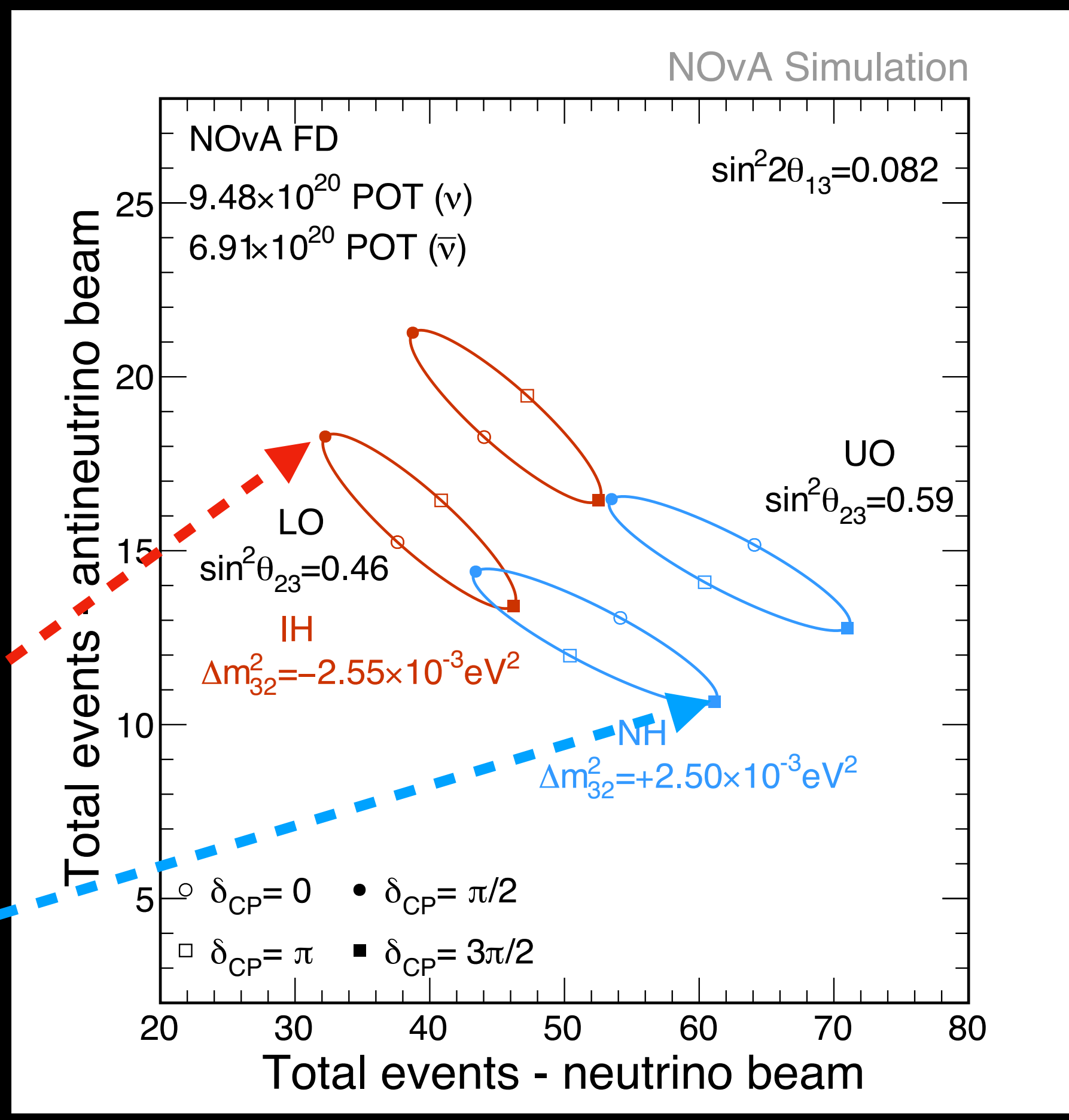
candidates, 30-75 $v_{\mathrm{e}}$ candidates 


\section{Predicting far detector spectra}
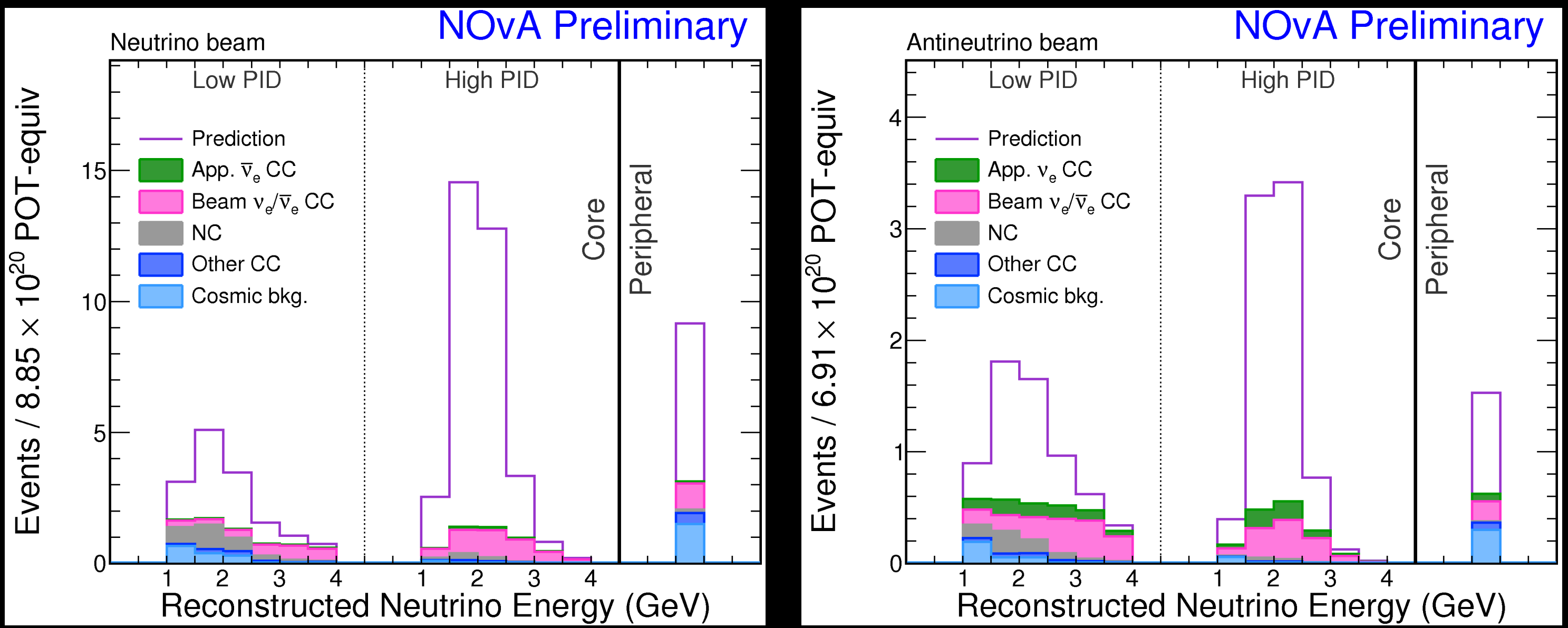

- An example for resulting prediction of candidates at far detector after constraining backgrounds with near detector and extrapolating oscillation signal prediction at a set of oscillation parameters

- Overall expectation varies based on oscillation parameters by changing the appearance signal and oscillated wrong-sign background

- Measured best-fit oscillation parameters will be the ones which give best agreement of overall expectation and the data (for both the ${ }^{\prime}{ }^{\prime}$ appearance and ${ }^{\prime}{ }^{\prime}{ }_{\mu}$ disappearance) 


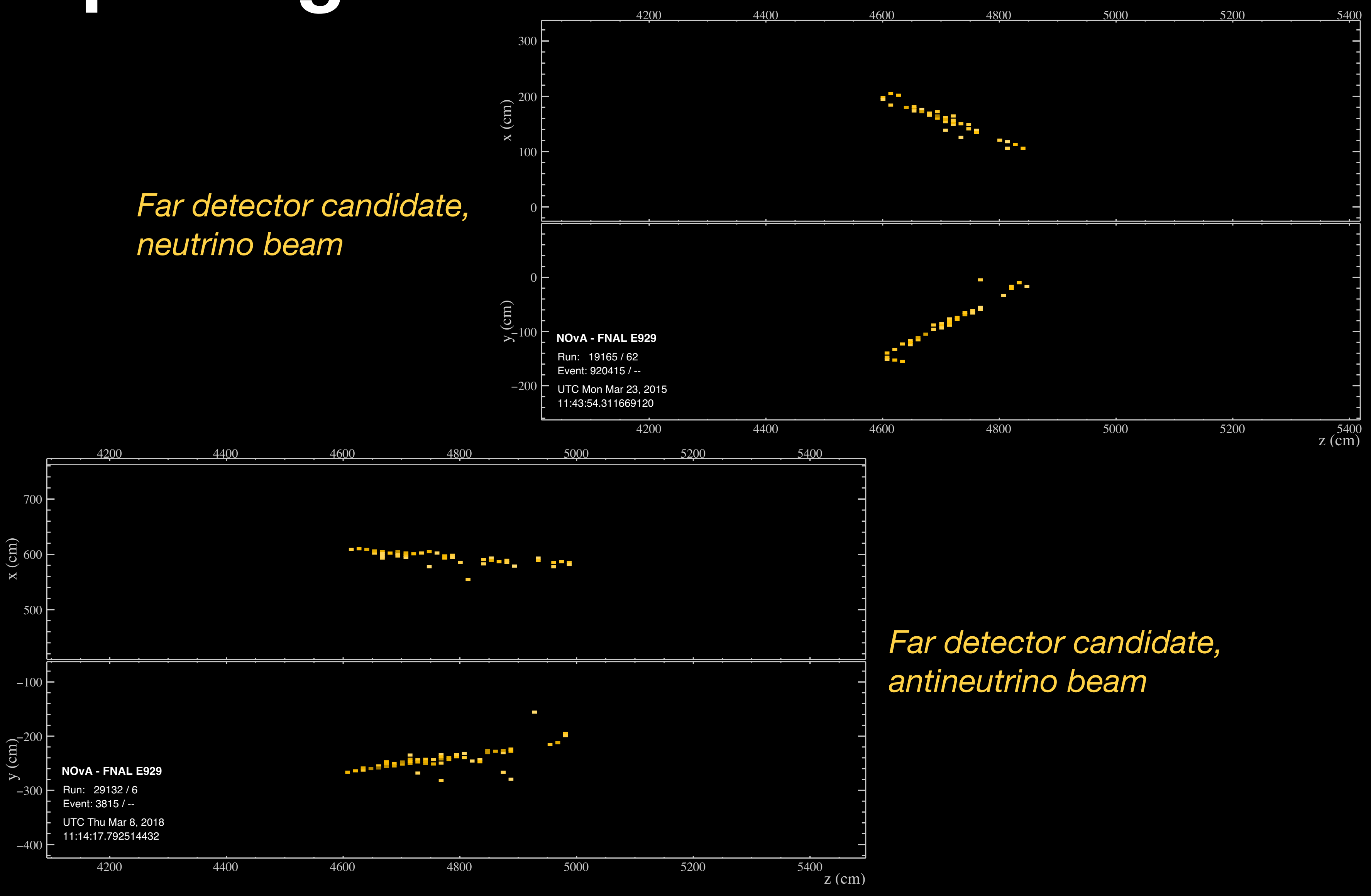




\section{$V_{e}, \overline{\mathbf{V}}_{\mathrm{e}}$ Results}

- In neutrino beam, select $\underline{58 v_{e} \text { candidates }}$

- Background prediction: 15.1 events

- $~ 12$ from beam (<1 wrong-sign oscillated) and $\sim 3$ cosmic origin

- In antineutrino beam, select $18 \bar{v}_{e}$ candidates

- Background prediction: 5.3 events

- Mostly of beam origin ( 1 wrongsign oscillated), $<1$ cosmic

- $>4 \sigma$ evidence for $\overline{\mathbf{v}}_{\mathrm{e}}$ appearancel

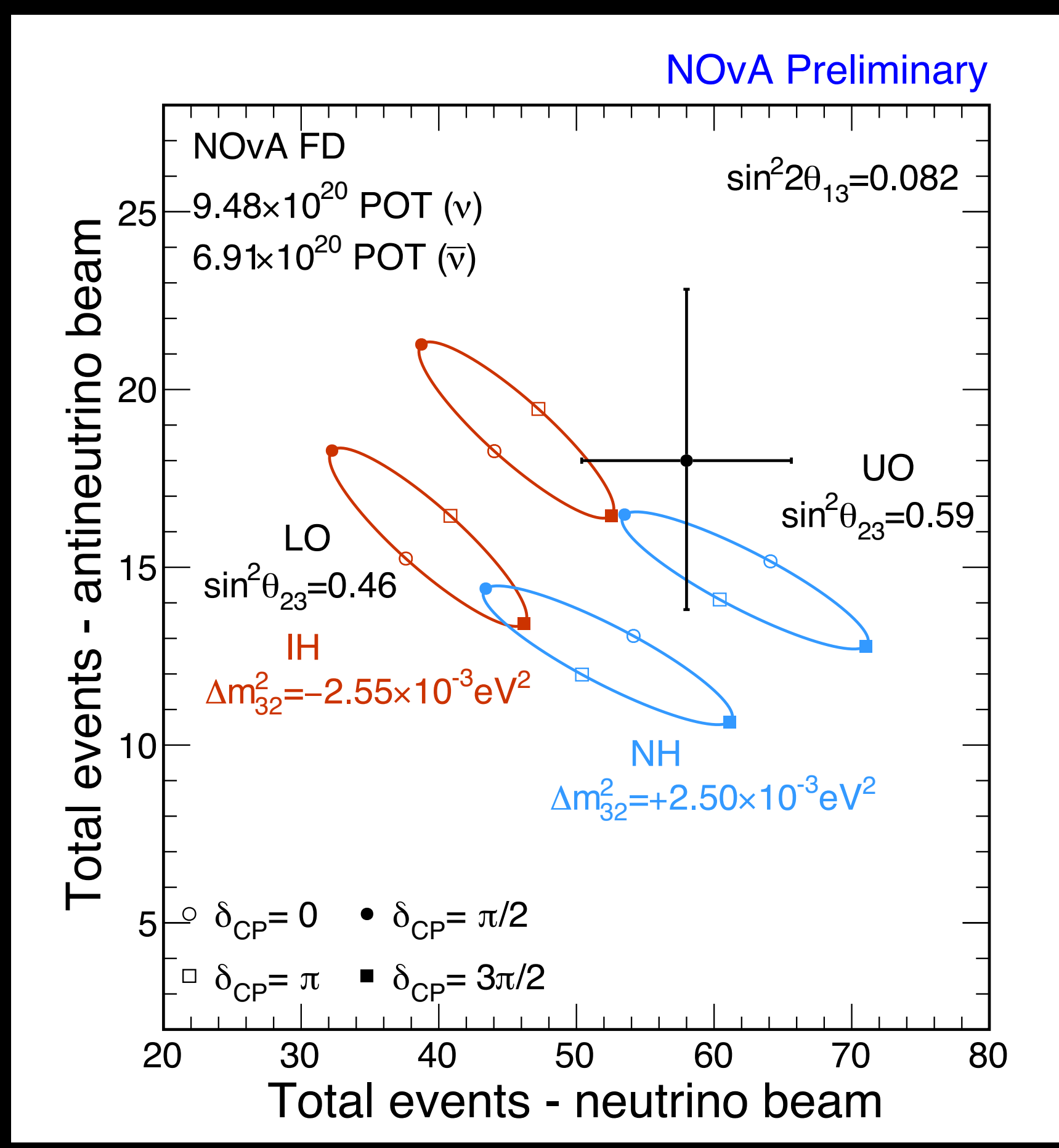




\section{$V_{e}, \overline{\mathbf{V}}_{\mathrm{e}}$ Results}

- In neutrino beam, select $\underline{58 v_{e} \text { candidates }}$

- Background prediction: 15.1 events

- 12 from beam (<1 wrong-sign oscillated) and $\sim 3$ cosmic origin

- In antineutrino beam, select $18 \bar{v}_{e}$ candidates

- Background prediction: 5.3 events

- Mostly of beam origin ( 1 wrongsign oscillated), $<1$ cosmic

- $>4 \sigma$ evidence for $\overline{\mathbf{v}}_{\mathrm{e}}$ appearance! Best fit prediction is for $59.0 v_{e}$ candidates and $15.9 \bar{v}_{e}$ candidates
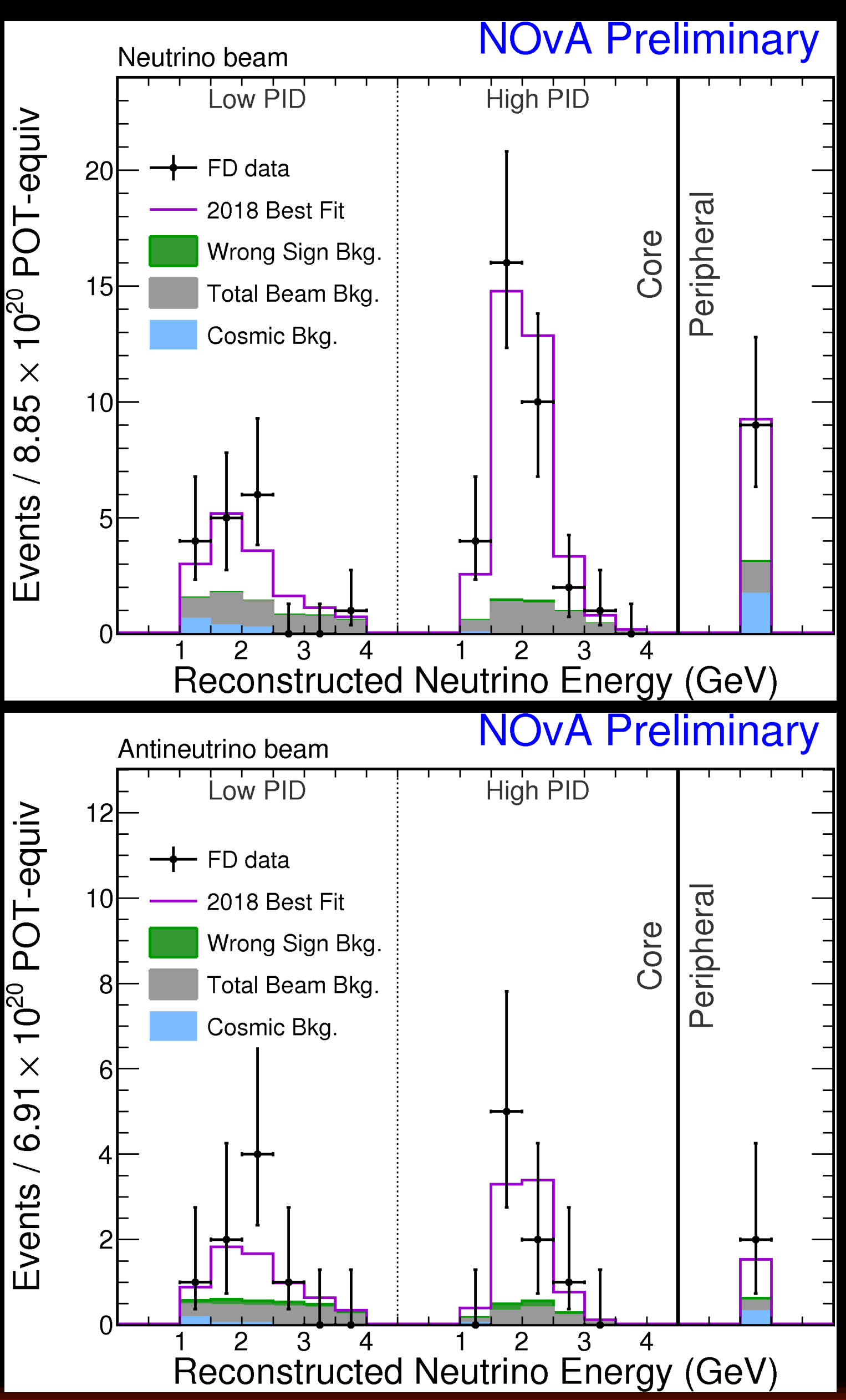


\section{Joint $\overrightarrow{\mathbf{v}}_{\mu}, \overrightarrow{\mathbf{v}}_{\mathbf{e}}$ Results}

- Joint analysis best fit prefers:

Normal hierarchy $\delta_{\mathrm{CP}}=0.17 \pi$

$\sin ^{2} \Theta_{23}=0.58 \pm 0.03$ $\Delta \mathrm{m}_{32^{2}}=\left(2.51^{+0.12}-0.08\right) \times 10^{-3} \mathrm{eV}^{2}$

Normal hierarchy preferred at $1.8 \sigma$ Exclude $\delta_{\mathrm{cP}}=\pi / 2$ in $\mathrm{IH}$ at $>3 \sigma$

Non-maximal mixing preferred at $\mathbf{1 . 8} \sigma$ Upper octant preference at similar level

- Full joint analysis requires statistical corrections via pseudo-experiment (Feldman-Cousins procedure)

- The story and importance of this procedure is the subject of next talk (D. Doyle)

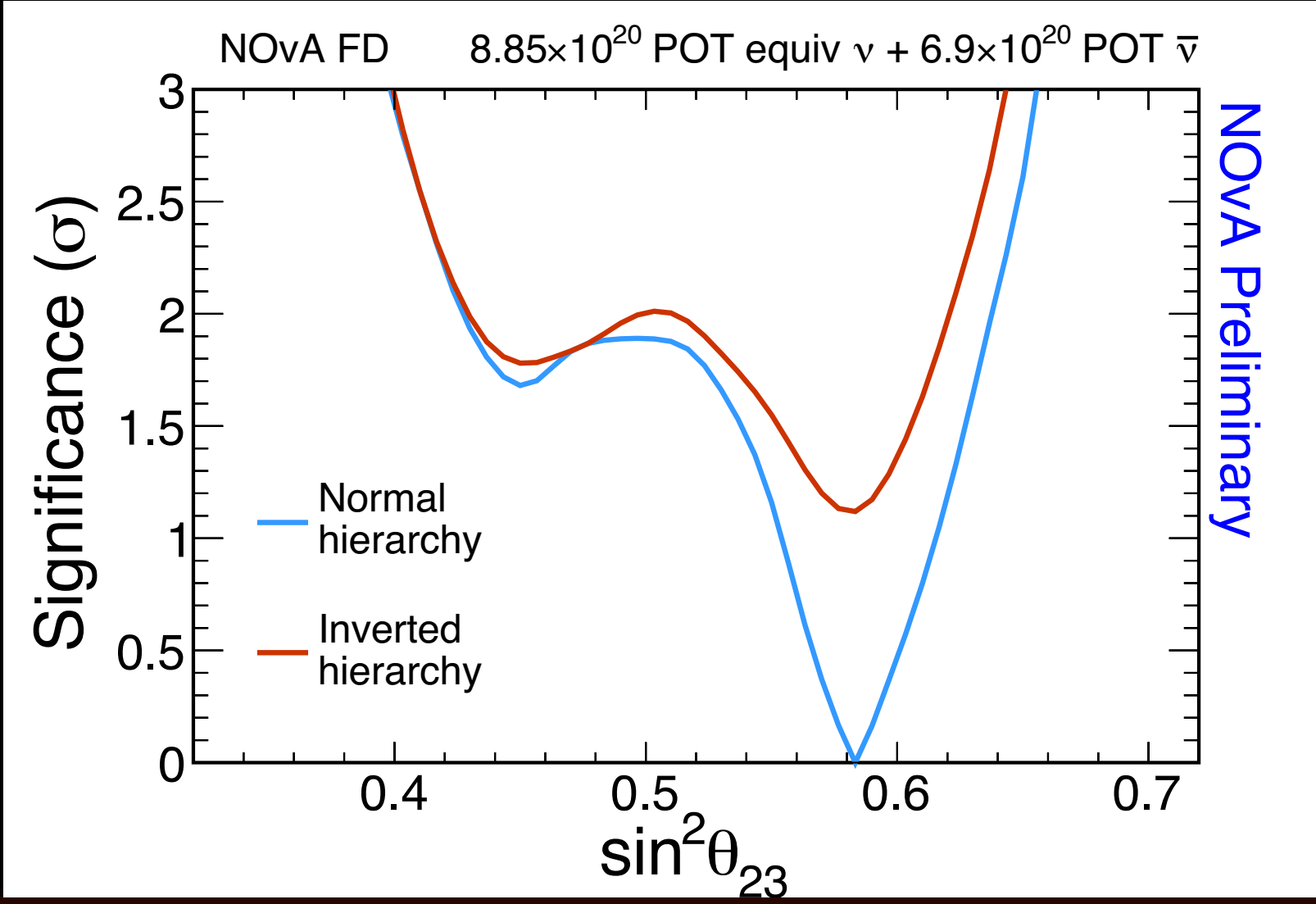

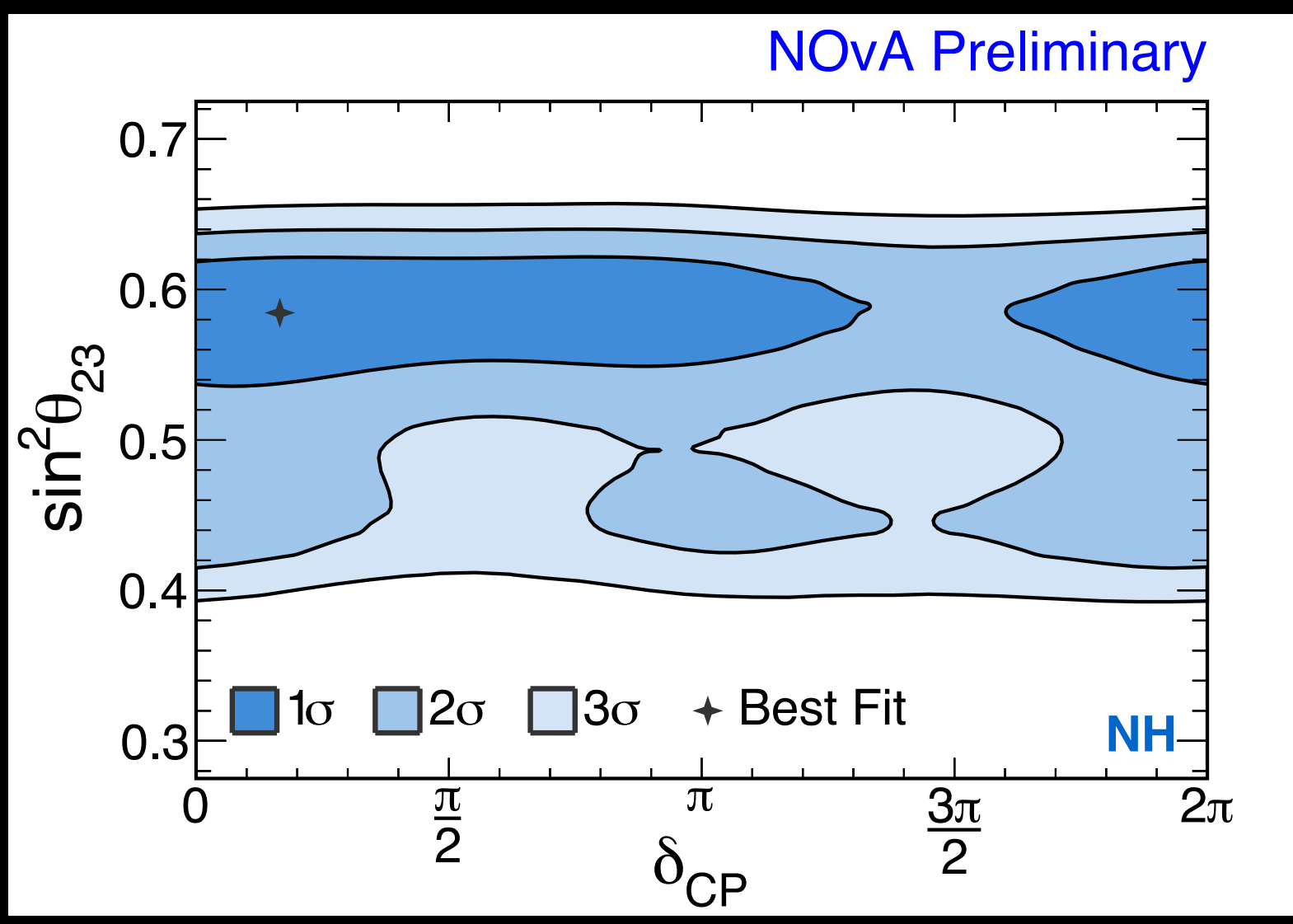

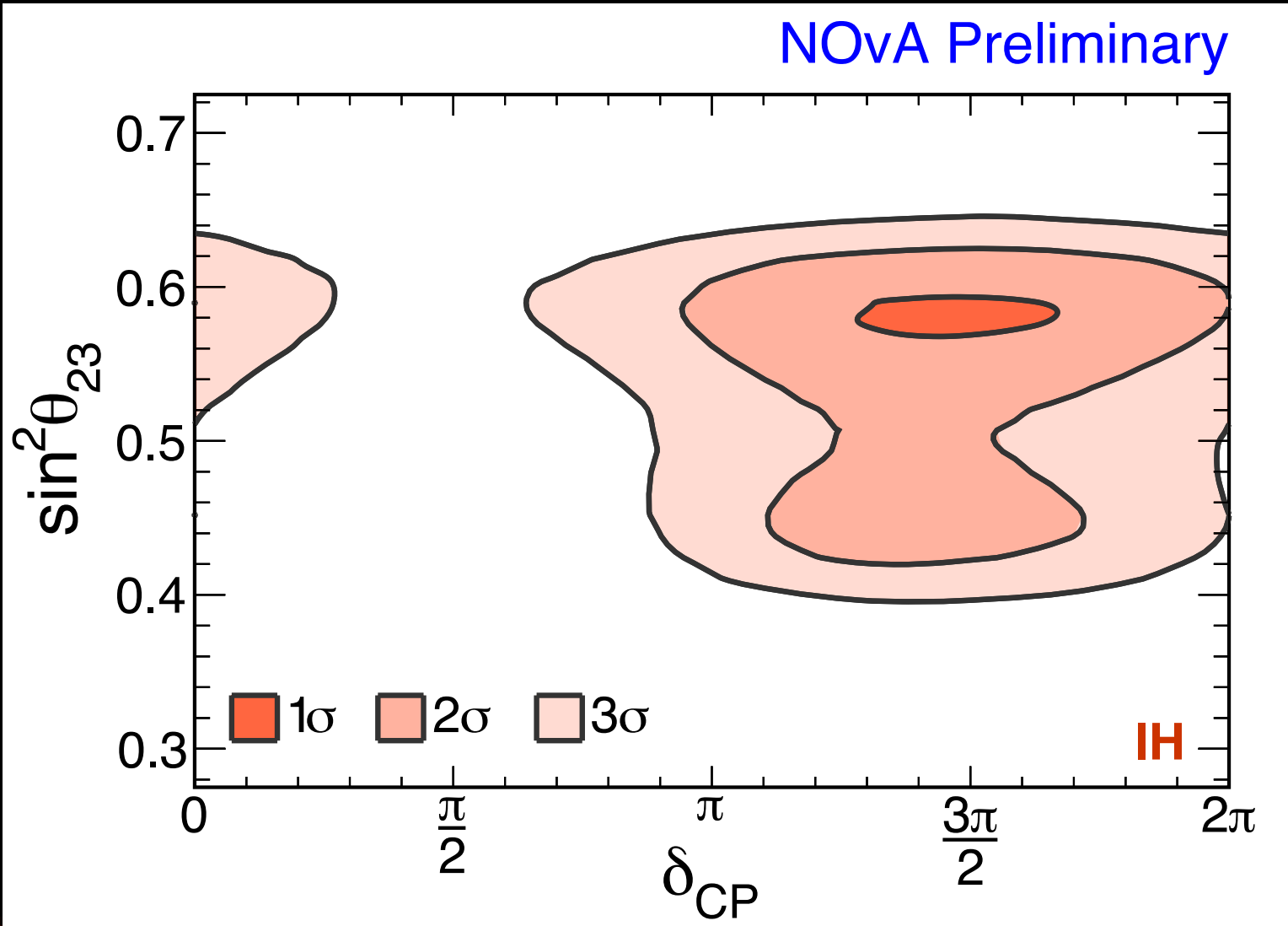




\section{Summary}

- Updated analysis now incorporates antineutrino beam for the first time (nearly $7 \times 10^{20}$ protons on target)

- Includes previous data set of nearly 9x1020 (full-detector equivalent) protons on target with neutrino beam

- First strong evidence $(>4 \sigma)$ for long-baseline $\bar{v}_{e}$ appearance in antineutrino beam!

- Achieved this in first $\overline{\mathrm{v}}$ analysis due to excellent beam performance

- Joint $\left(\vec{v}_{\mu} \text { disappearance and }{ }^{\prime}\right)_{e}$ appearance analysis has slight preference towards normal mass hierarchy and non-maximal $\Theta_{23}$ [upper octant]

- Further data and analysis upgrades are expected to provide further sensitivity to key oscillation parameters in the coming years 


\section{Backup}

@ II 


\section{Neutrino Oscillation}

H. Nunokawa, S. Parke, J. W. F. Valle. "CP Violation and neutrino oscillations." Prog.Part.Nucl.Phys., 60 (2008) 338-402

$$
\begin{aligned}
P\left(v_{\mu} \rightarrow V_{e}\right) & =\sin ^{2} \Theta_{23} \sin ^{22} \Theta_{13} \frac{\sin ^{2}\left(\Delta_{31}-\mathrm{aL}\right)}{\left(\Delta_{31}-\mathrm{aL}\right)^{2}} \Delta_{31}{ }^{2} \\
& +\sin 2 \Theta_{23} \sin 2 \Theta_{13} \sin 2 \Theta_{12} \frac{\sin \left(\Delta_{31}-\mathrm{aL}\right)}{\left(\Delta_{31}-\mathrm{aL}\right)} \Delta_{31} \frac{\sin (\mathrm{aL})}{(\mathrm{aL})} \Delta_{21} \cos \left(\Delta_{31}+\delta\right) \\
& +\cos ^{2} \Theta_{23} \sin ^{22} \Theta_{12} \frac{\sin ^{2}(\mathrm{aL})}{(\mathrm{aL})^{2}} \Delta_{21}{ }^{2}
\end{aligned}
$$

To first order, where

$$
\begin{aligned}
\Delta \Delta_{i j} & =\Delta m_{i j}{ }^{2} L / 4 E \\
a & =G_{F} N_{e} / \sqrt{ } 2
\end{aligned}
$$

And in the case of antineutrinos, the sign of the CP violating phase $(\delta)$ and sign of "a" flip. 


\section{Recall $\overrightarrow{\mathbf{v}}_{\boldsymbol{\mu}}$ Result}

- Details in D. Torbunov talk

- Select $113 v_{\mu}$ candidates in neutrino beam and $65 \bar{v}_{\mu}$ candidates in antineutrino beam

- Expecting $730+38_{-49} \mathrm{~V}_{\mu}$ candidates in neutrino beam and $266^{+12}-14 \overline{\mathrm{V}}_{\mu}$ candidates in antineutrino beam, in case of no oscillations

- Cosmic background prediction 2 events in neutrino beam and $<1$ event in antineutrino beam

- The results of the $v_{\mu}$ and $\bar{v}_{\mu}$ disappearance and the $v_{e}$ and $\bar{v}_{e}$ appearance are then studied in a joint analysis and result in a joint oscillation fit in 3 neutrino paradigm
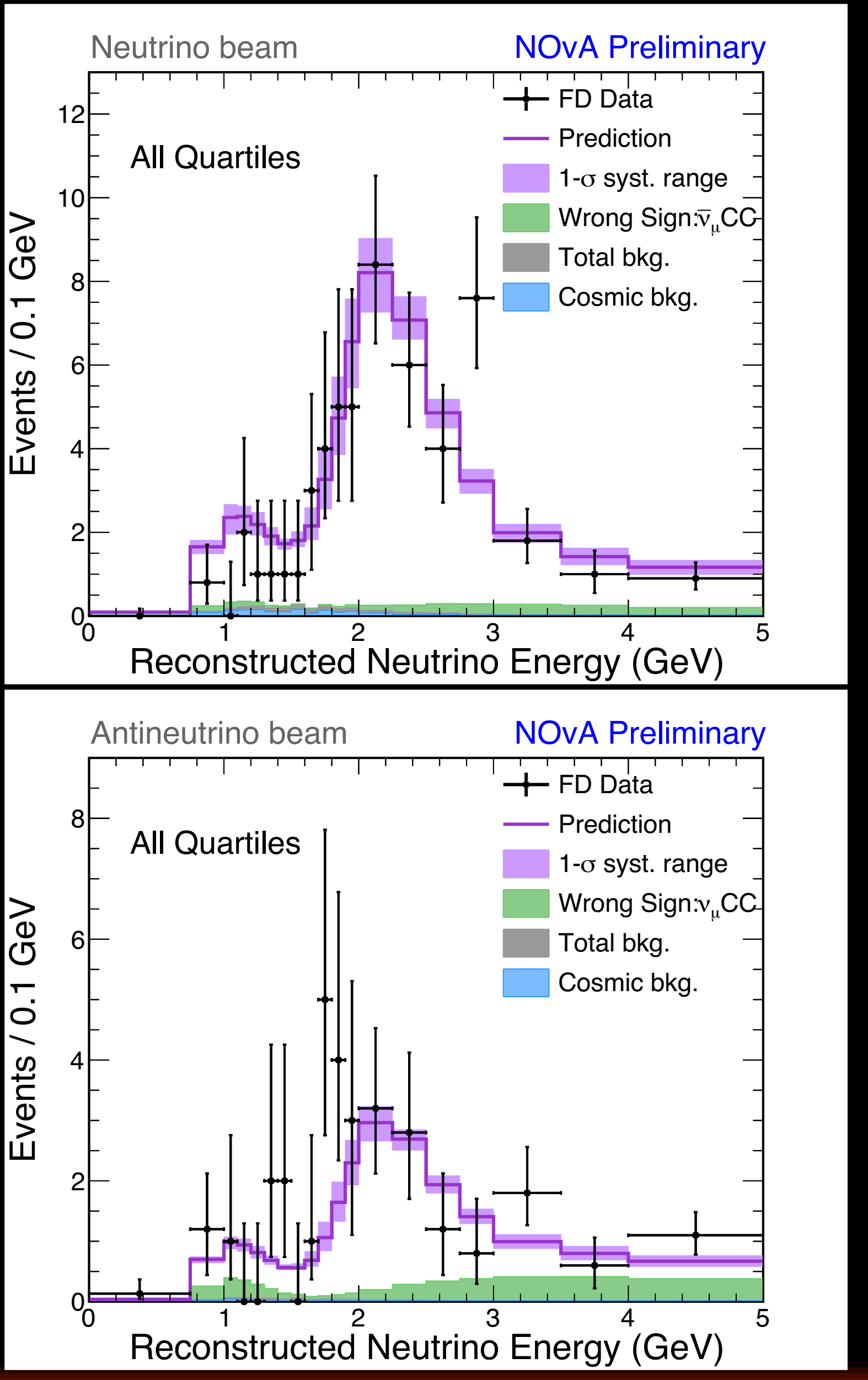


\section{Recall $\vec{v}_{\mu}$ Result}

- Select $113 \mathrm{v}_{\mu}$ candidates in neutrino beam and $65 \overline{\mathrm{v}}_{\mu}$ candidates in antineutrino beam

- Expecting $730^{+38}-49 \mathrm{~V}_{\mu}$ candidates in neutrino beam and $266^{+12}-14 \overline{\mathrm{V}}_{\mu}$ candidates in antineutrino beam, in case of no oscillations
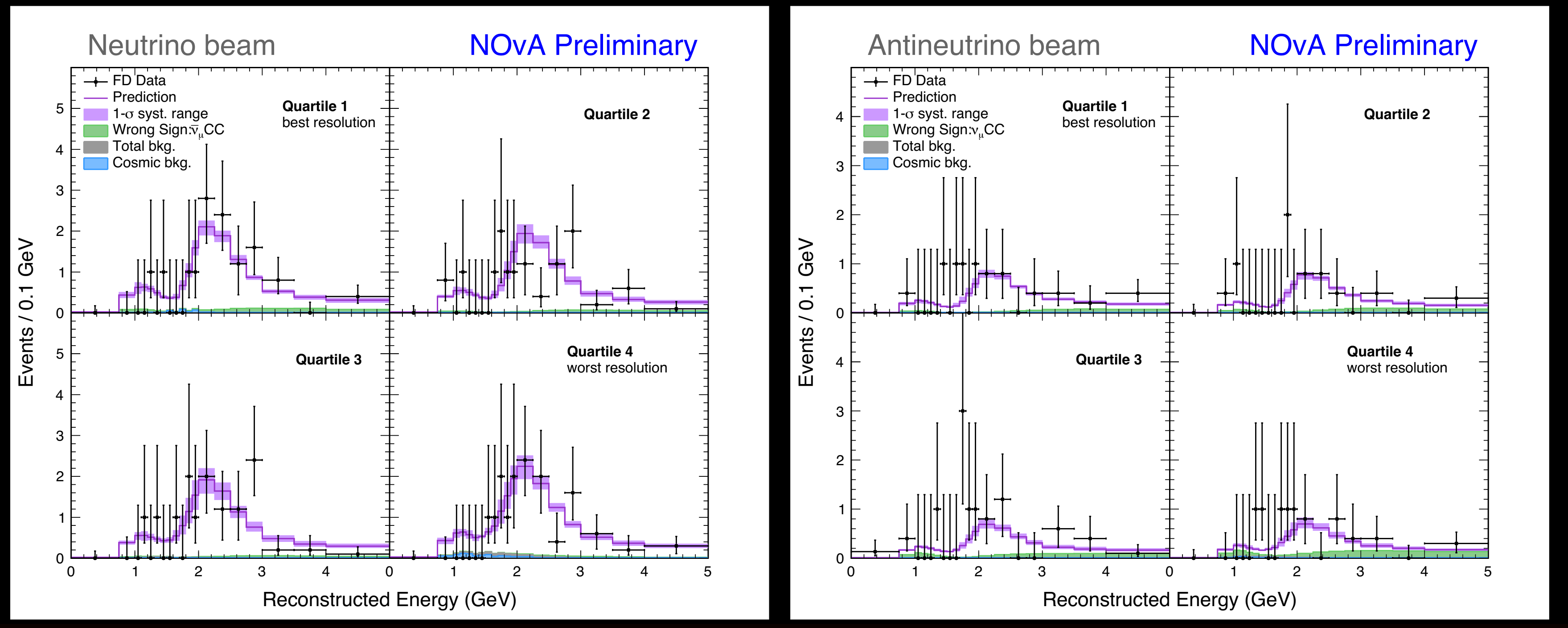


\section{Future sensitivity}

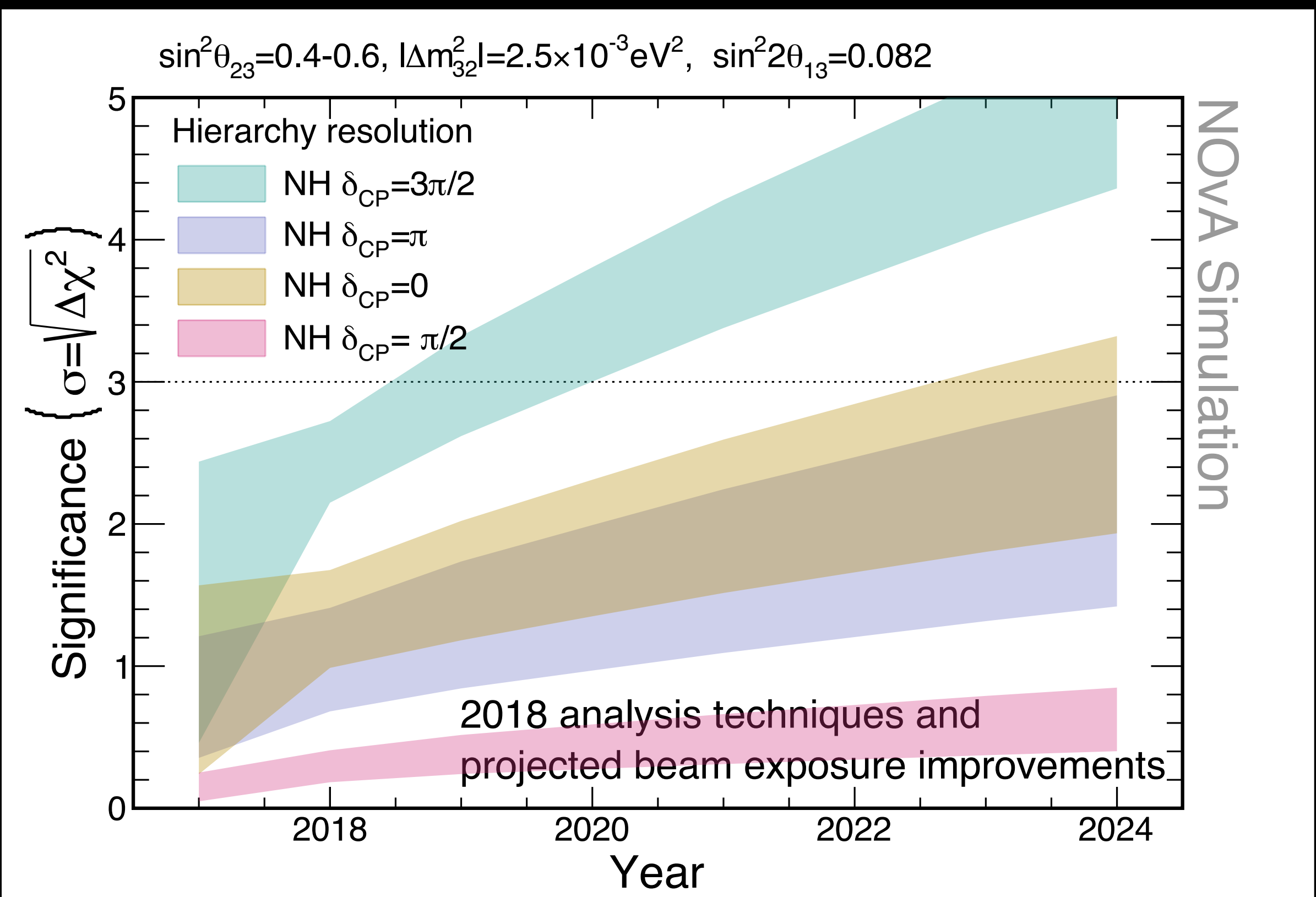

Mass hierarchy sensitivity 30 by 2020 for most favorable parameters. Can reach 30 by 2024 for wider range of parameters. 


\section{Event selection for $v_{e}+\bar{v}_{e}$}

- Details of reconstruction detailed in talk by M. Groh

- NOvA uses CVN (Convolutional Visual Network) to perform event classification based on final state topologies

- Select events $\mathrm{w} / \mathrm{V}_{\mathrm{e}}\left(\overline{\mathrm{V}}_{\mathrm{e}}\right)$ classifier score \& reconstructed information indicating likely $v_{e}\left(\bar{V}_{e}\right)$ candidate

- Near detector and far detector selection broken into two subcategories based on CVN electron score.

- Far detector has further peripheral sample with larger cosmic background but recovers appearance signal

- Energy estimator for $\mathrm{v}_{\mathrm{e}}, \overline{\mathrm{V}}_{\mathrm{e}}$ characterizes energy deposits as coming from EM activity (or not) utilizing CVN on specific prongs. Estimator is 2nd-order polynomial in EM energy and non-EM energy

- $11 \% \mathrm{E}$ resolution in neutrino beam, $9 \%$ antineutrino

- Energy range of interest $0-4.5 \mathrm{GeV}$ in near detector, $1-4 \mathrm{GeV}$ in far detector (0-4.5 GeV in peripheral)
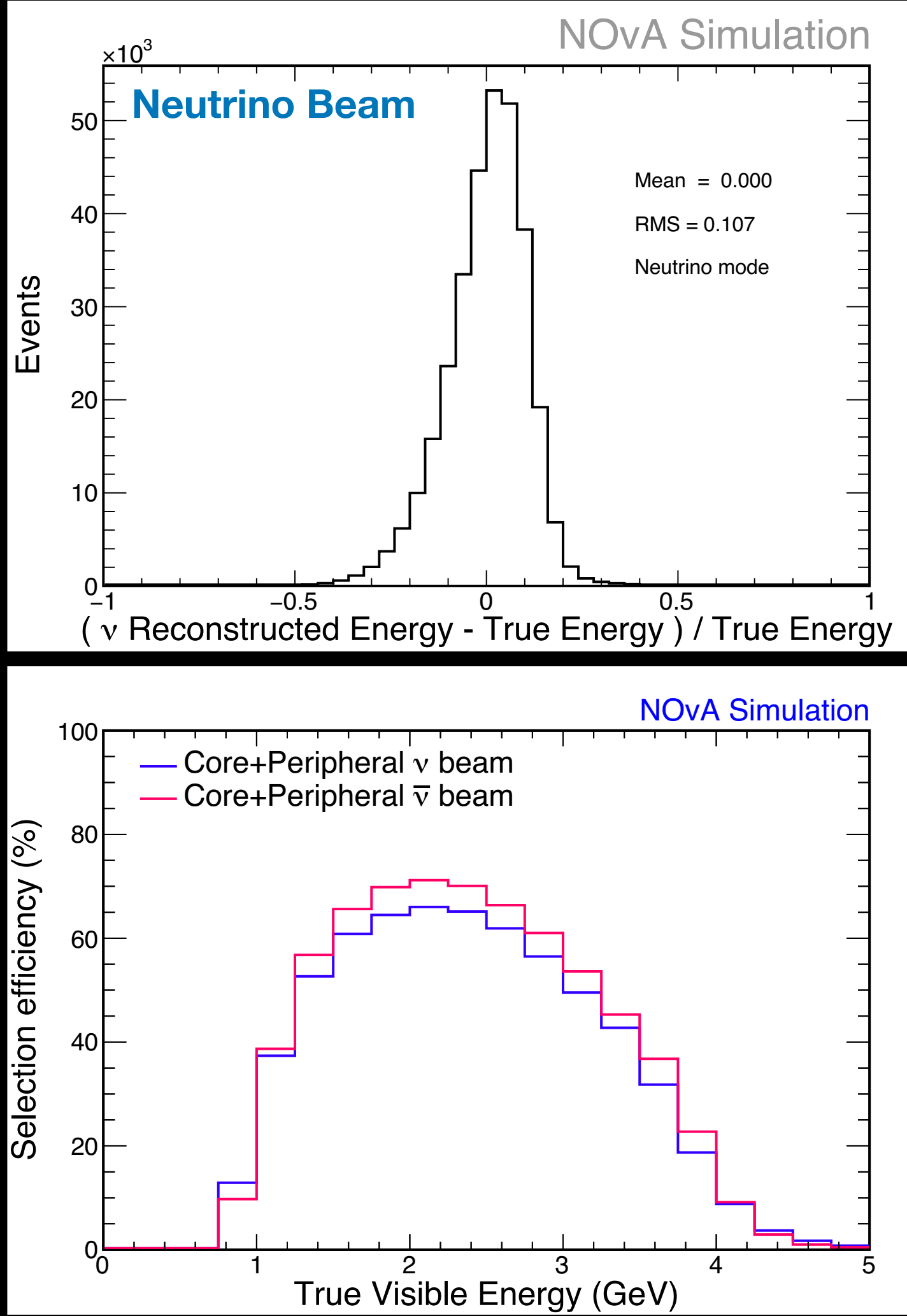


\section{Event selection Cross-checks}

- Muon-removed electron (MRE) events replace muons in selected near detector $\mathrm{v}_{\mu}$ data candidates with electron showers of same momentum

- Takes advantage of hadronic components directly from data

- Electron shower simulation

- Probes understanding of selection efficiency for $v_{e}$ events
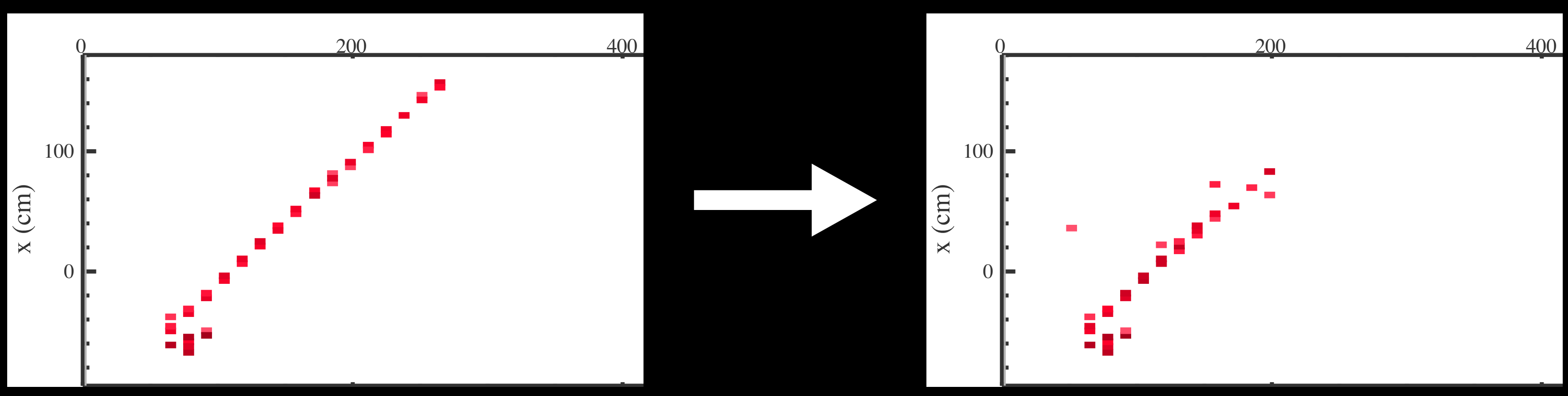

Example 


\section{Event selection Cross-checks}

- Muon-removed bremsstrahlung (MRBrem) removes muons from far detector cosmic data and keeps the bremsstrahlung electron shower

- Probes understanding of selection efficiency for $v_{e}$ events

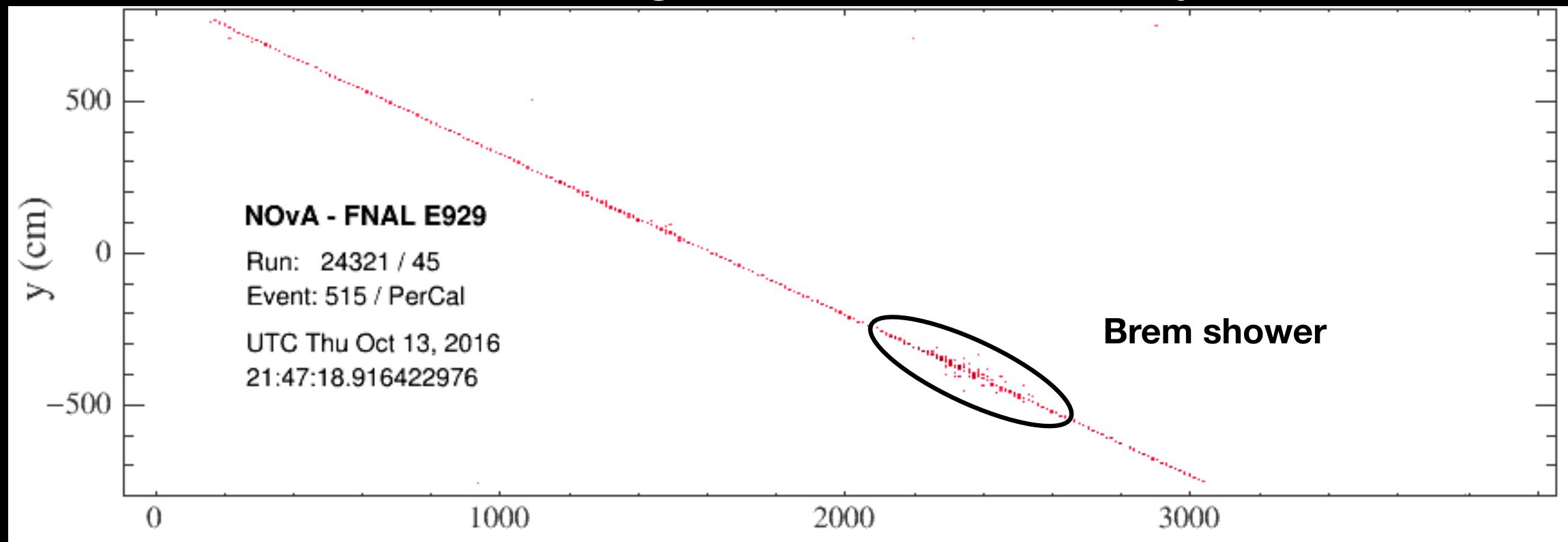

Example

NOVA - FNAL E929

Run: $24321 / 45$

Event: $515 /$ PerCal

UTC Thu Oct 13, 2016

21:47:18.916422976

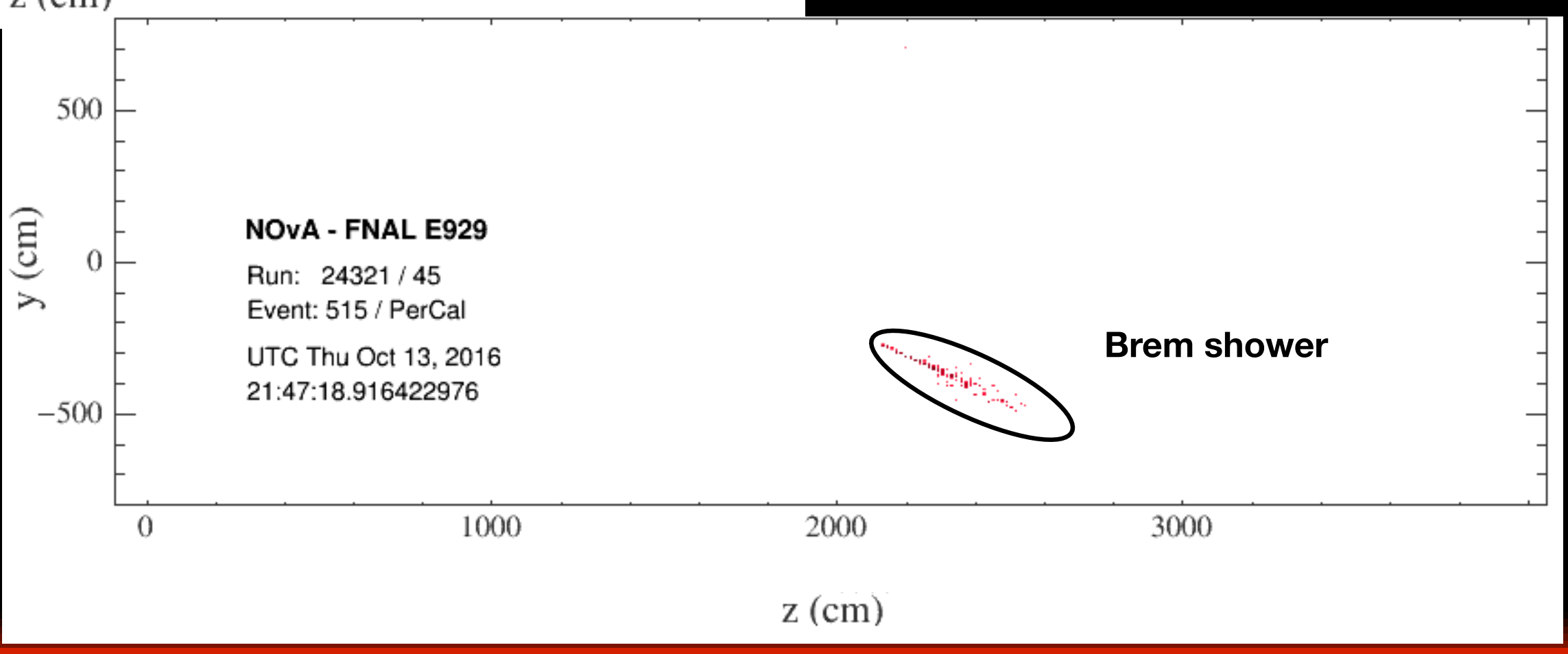




\section{Event selection Cross-checks}

- Muon-removed bremsstrahlung (MRBrem) removes muons from far detector cosmic data and keeps the bremsstrahlung electron shower

- Probes understanding of selection efficiency for $v_{e}$ events
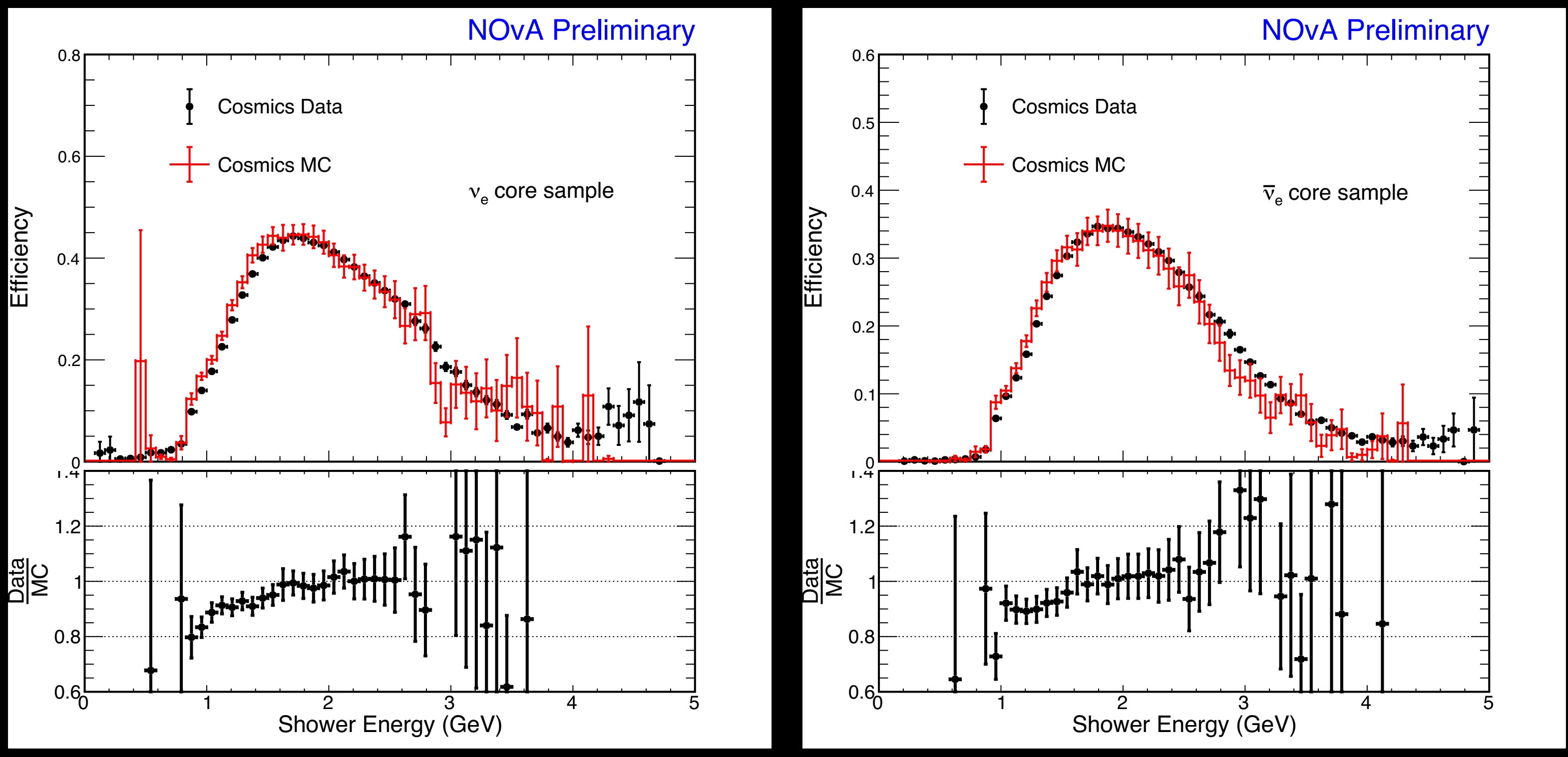


\section{NOvA flux}

- For $v_{e}$ appearance analysis, characterization of the $v_{\mu}$ beam (which oscillates to signal) and the inherent $v_{e}$ background component in the beam are important
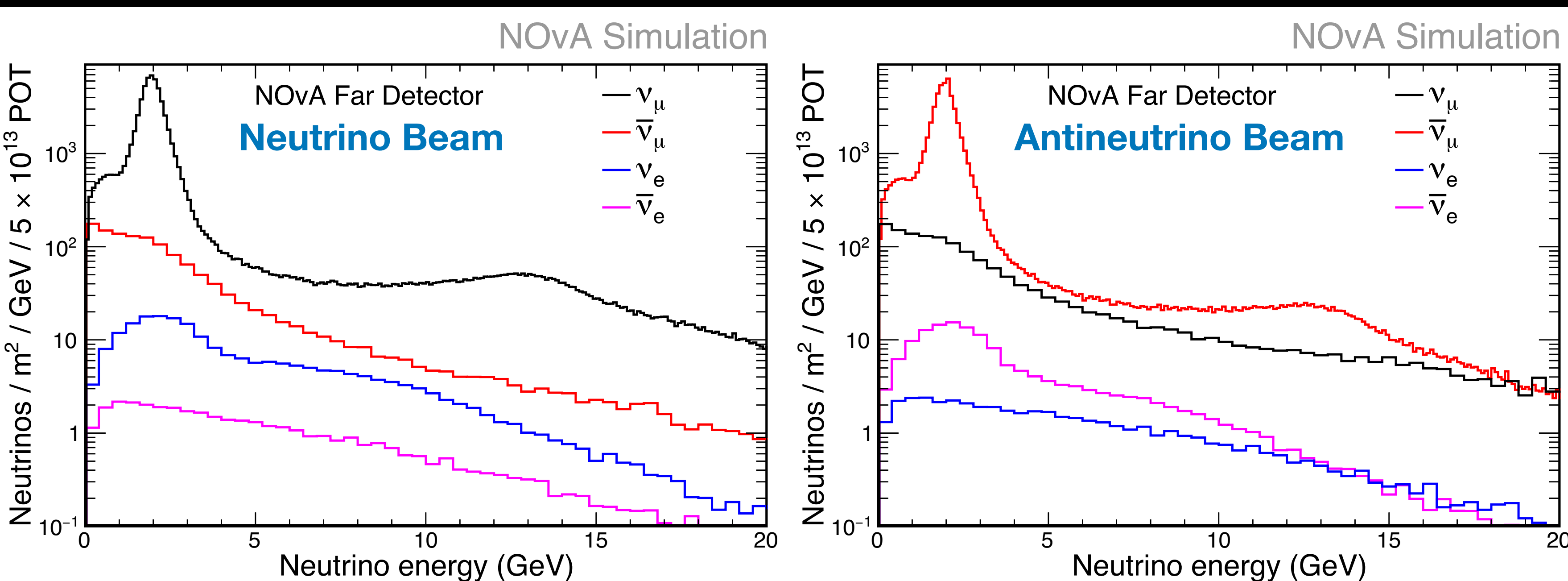


\section{Predicting far detector spectra}

- Since NOvA has functionally similar near and far detector, extrapolation procedure reduces a number of systematic effects

- Makes flux uncertainties quite small; greatly reduces cross-section uncertainties

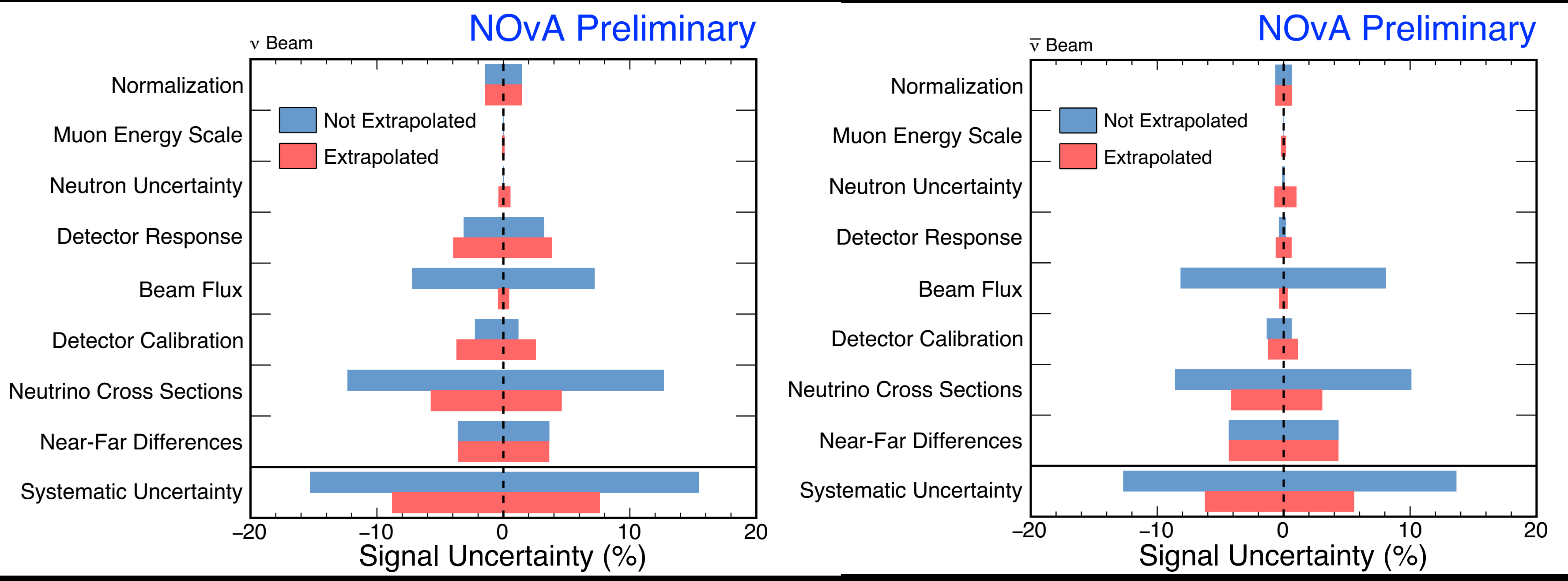




\section{Joint $\overrightarrow{\mathbf{v}}_{\mu}, \overrightarrow{\mathbf{v}}_{\mathbf{e}}$ Results}

- Joint analysis is statistics limited in the major oscillation parameter results $\left(\sin ^{2} \Theta_{23}, \Delta m_{32^{2}}, \delta_{C P}\right)$
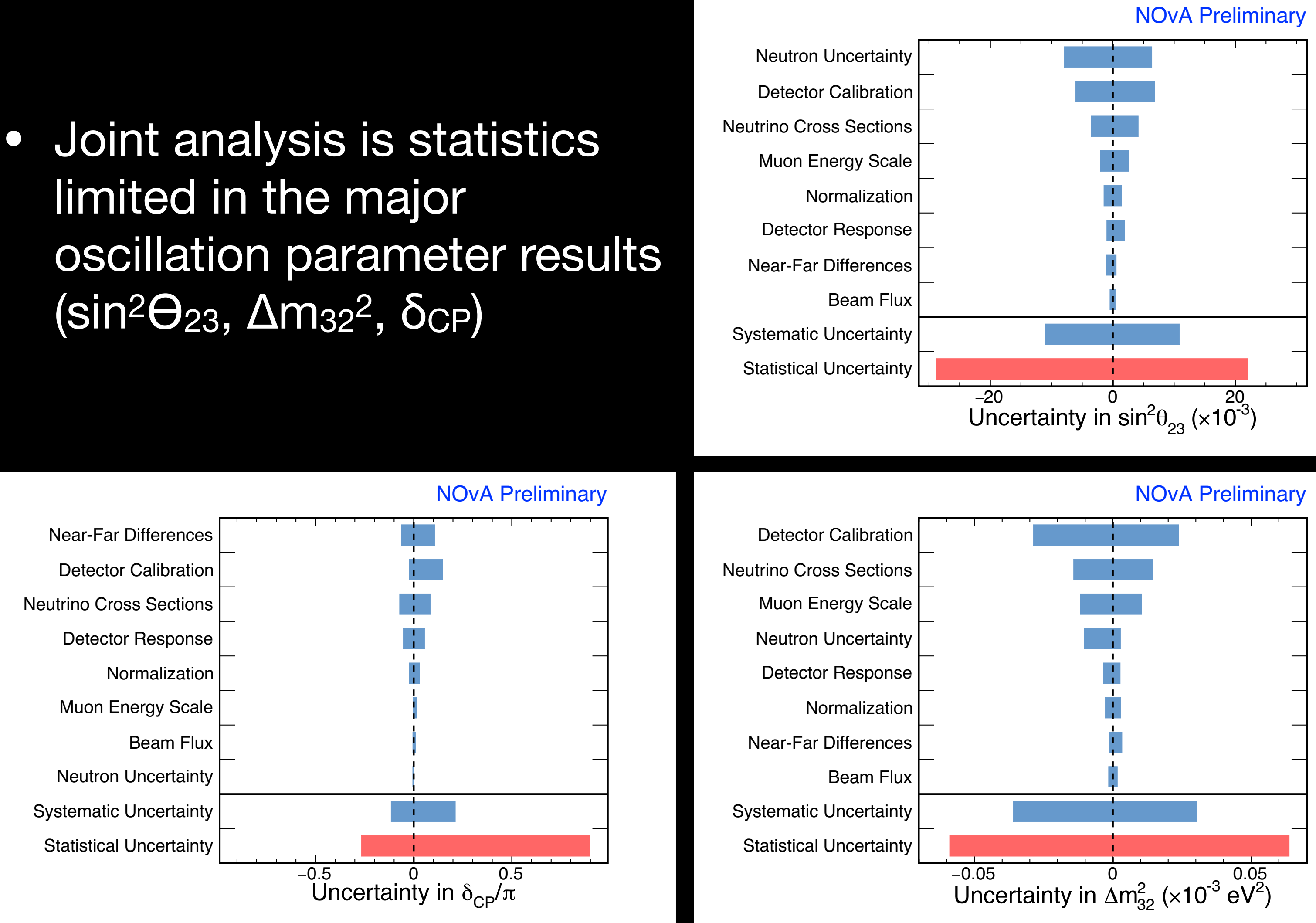


\section{Neutron systematic}

- Scale amount of energy deposited by some neutrons to cover the discrepancy at low energy

- Shifts mean $v_{\mu}$ energy by $1 \%$ in antineutrino beam and $0.5 \%$ in neutrino beam

- Resolution changed by fractions of a percent

- Negligible impact found on selection efficiency

(
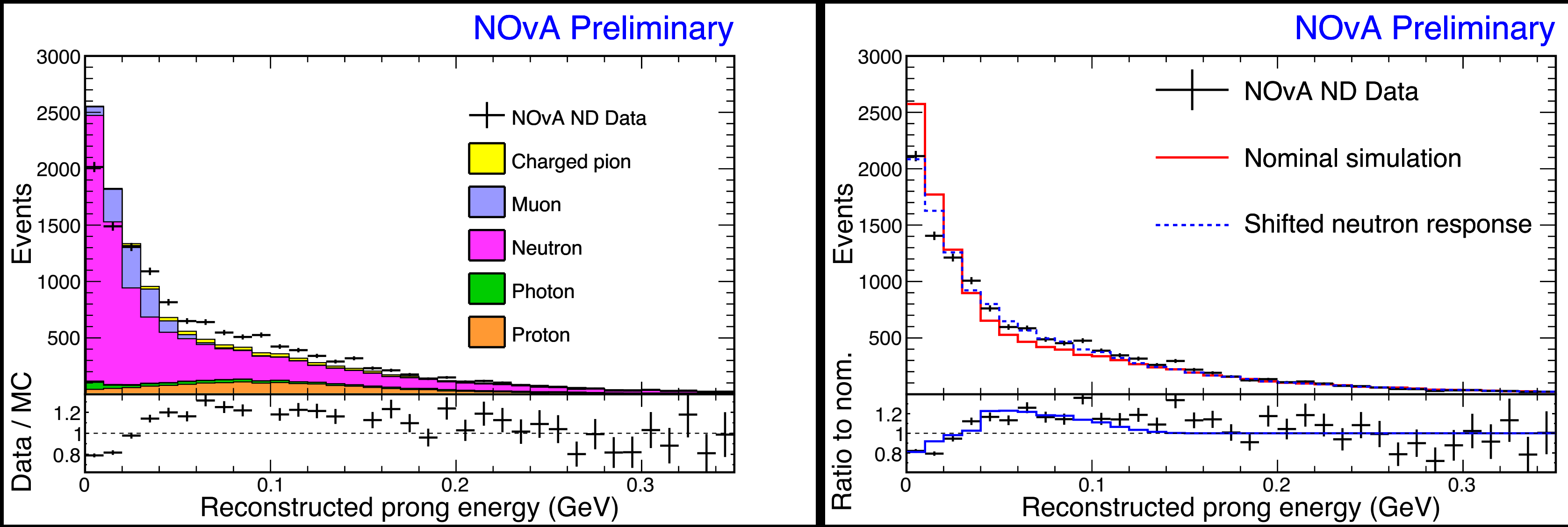